Check for updates

Cite this: Phys. Chem. Chem. Phys.,

2021, 23, 3883
Received 23rd November 2020, Accepted 28th January 2021

DOI: $10.1039 / \mathrm{d} 0 \mathrm{cp} 06073 a$

rsc.li/pccp

\title{
First-principles calculation of ${ }^{11} \mathrm{~B}$ solid-state NMR parameters of boron-rich compounds II: the orthorhombic phases $\mathrm{MgB}_{7}$ and $\mathrm{MgB}_{12} \mathrm{C}_{2}$ and the boron modification $\gamma-\mathrm{B}_{28}^{\dagger}$
}

\author{
Martin Ludwig and Harald Hillebrecht (D)*
}

\begin{abstract}
Based on the work on referencing ${ }^{11} \mathrm{~B}$ nuclear magnetic resonance (NMR) spectra for molecular icosahedral boranes and the subsequent transfer to the rhombohedral boron-rich borides of the $\alpha-r_{12}$ type, we show that the magic angle spinning (MAS) NMR spectra of boron-rich borides with four or five symmetry-independent boron atoms can also be calculated. The calculations are performed on the level of density functional theory (DFT) using the gauge-including projector-augmented wave (GIPAW) approach. As model compounds $\mathrm{O}-\mathrm{MgB}_{12} \mathrm{C}_{2}$ and $\mathrm{MgB}_{7}$ are used, for which the experimental spectra could be calculated in excellent agreement with a deviation of 1 to $2 \mathrm{ppm}$. Based on the calculations, the different $B$ atoms can be assigned to the respective signals, taking into account the quadrupolar coupling constants $C_{q}$ from computation of the electric field gradient (EFG) with its main axis $V_{z z}$. It is shown that due to the specific geometric conditions of icosahedra, the magnitudes of $V_{z z}$ for the boron atoms involved in exohedral B-B bonds to neighbouring icosahedra depend only on the valence electron density of the bond critical point and the distance. This also applies to the bonds to the interstitial $\mathrm{B}_{2}$ unit in $\mathrm{MgB}_{7}$, but not to bonds to the heteroatom of the $\mathrm{C}_{2}$ dumbbell in o- $\mathrm{MgB}_{12} \mathrm{C}_{2}$. Both results are in line with our previous observations for the rhombohedral species $\left(\alpha-r B_{12} ; B_{12} X_{2}\right.$ with $\left.X=P, A s, O\right)$. Finally, the spectrum of $\gamma-B_{28}$ was calculated, whose structure also contains $B_{12}$ icosahedra and interstitial $B_{2}$ dumbbells. Here, a very similar bonding situation is found for the icosahedron, but the calculations show that the situation for the $\mathrm{B}_{2}$ unit is clearly different. In general, the only parameter that needs to be varied to fit calculated and measured spectra is the linewidth, as this cannot be calculated. For the cases of $\mathrm{O}-\mathrm{MgB}_{12} \mathrm{C}_{2}$ and $\mathrm{MgB}_{7}$ signal areas are related to corresponding site multiplicities. A prerequisite for the successful application of the chosen method seems to be the presence of a semiconductor with a sufficiently large band gap, which is the case for the compounds investigated.
\end{abstract}

\section{Introduction}

Due to its distinctive elemental structures, bonding mode and physical properties, B raises questions regarding not only its elemental structures but also the compounds derived from them, such as boron-rich borides. To this date, the exact composition of solid phases and the resulting structure-property relationships are a challenge for both experimentalists and theoreticians. ${ }^{1}$

Being an element with noteworthy electron deficit, this element is known to form numerous deltahedral structures such as octahedra or icosahedra. In fact, it is precisely for this

Institut für Anorganische und Analytische Chemie, Albert-Ludwigs-Universität, Albertstr. 21, 79104 Freiburg, Germany. E-mail: harald.hillebrecht@ac.uni-freiburg.de $\dagger$ Electronic supplementary information (ESI) available. See DOI: 10.1039/ d0cp06073a reason that $\mathrm{B}$ confronts us with certain puzzles, to which the solutions may not only provide an adequate description of interesting properties but often also fertile ground for concept development in chemistry. For example, early work of LonguetHiggins $^{2,3}$ on the electronic structure of molecular B closoclusters paved the way for the electron counting rules of Wade, ${ }^{4}$ which became a well-known part of common chemistry textbooks. Nevertheless, the investigation of the bonding situation of $\mathrm{B}$ polyhedra in molecules ${ }^{5}$ and especially in solid compounds $^{6-8}$ is still of essential importance.

The structural determination of the modification $\gamma-\mathrm{B}\left(\gamma-\mathrm{B}_{28}\right),{ }^{9,10}$ in which icosahedral building units are linked to each other and to $\mathrm{B}_{2}$ dumbbells, has stimulated a vivid discussion about the bonding situation prevailing there. ${ }^{11-16}$ Still, current work is examining the structural influences on problems of the hardness description for this compound. ${ }^{17}$ But also for binary borides such as $\alpha-\mathrm{SiB}_{3-x}$, the distribution of $\mathrm{Si}$ in the condensed polyhedra of 
B is of ongoing interest for an understanding of corresponding physical properties. ${ }^{18}$

Nuclear magnetic resonance (NMR) spectroscopy is a microscopic tool to study the electronic structure in numerous boron compounds. Because the nuclear spin $I$ of both naturally occurring isotopes ${ }^{10} \mathrm{~B}$ and ${ }^{11} \mathrm{~B}$ is greater than one-half, the $\mathrm{B}$ nucleus interacts with the electronic field gradient (EFG). In addition to the Zeeman effect, this generated quadrupole moment leads to a further splitting of the energy levels in the magnetic field. The internuclear couplings, that are the dipolar coupling through space and the $J$ coupling through bonds, generally show several orders of magnitude less contributions in solid-state NMR (SSNMR) spectra of B. ${ }^{19}$

The orientation-dependent contributions of many of the mentioned interactions are relevant for the recorded signal shape in SSNMR and can hamper the interpretation of measurements by spectral broadening. These anisotropic components disappear under magic angle spinning (MAS) conditions, that means the sample is physically rotated around an axis which inclines the so called magic angle relative to the external magnetic field. However, even with this approach, a contribution of the quadrupolar coupling remains in second-order and can make unambiguous processing difficult, notably if more than one species is detected in the spectrum. ${ }^{20}$ Accordingly, the MAS NMR spectra of quadrupolar B nuclei are not only influenced by the chemical shift but also by the quadrupolar coupling, which also affects the observed shifts of the central transition $(1 / 2 \leftrightarrow-1 / 2)^{21,22}$ and influences sideband patterns $( \pm 3 / 2 \leftrightarrow \pm 1 / 2)$ as well as the profile via the anisotropy parameter $\eta_{\mathrm{q}}$.

Apart from the problem that overlapping signals of several sites may complicate spectra analysis, it is very difficult to guarantee phase purity in case of solid compounds of B. The element is known to incorporate small impurities such as C. This sensitivity leads to an extraordinary difficulty in the synthesis of samples with the requirement for special purity or stoichiometry. ${ }^{16,23}$ An example is the study ${ }^{24}$ on the existence of the tetraboride $\mathrm{CaB}_{4}$. In particular, the $\mathrm{C}$ content of $\mathrm{CaB}_{4-x} \mathrm{C}_{x}$ was found to be rather low $(<5 \%)$ but its presence turned out to be essential for the synthesis. Of the three different crystallographic positions of boron, only one could be clearly assigned a chemical shift and quadrupole coupling constant from the measured NMR spectra, since the other two showed side band patterns of low intensity. Finally, we would like to point out that deviations from the actual solid phase structure due to impurities can also lead to errors in processed parameters.

A combination of experimental data with calculations on an $a b$ initio level has been proven efficient in this context. Especially in the case of borides the electronic structure could be related to results for quadrupole couplings measured by NMR techniques, since this observable can be derived directly from the electron density distribution. The EFG components for $\mathrm{MB}_{12}(\mathrm{M}=\mathrm{Y}, \mathrm{Zr}, \mathrm{Lu})$ were calculated with cuboctahedral units ${ }^{25}$ and later the magnitude and orientation of $\mathrm{YB}_{12}$ were confirmed by measurements. ${ }^{23}$ In addition, computational studies ${ }^{26-29}$ on metal borides mainly consisting of $\mathrm{B}_{6}$ octahedra show good agreement between theory and experiment. And as recent studies show, ${ }^{30}$ such calculations of the EFG parameters still help to unveil MAS NMR investigations on the ternary borides $\mathrm{YTB}_{4}(\mathrm{~T}=\mathrm{Mo}, \mathrm{W}, \mathrm{Re})$ with a planar $2 \mathrm{D}$ net of $\mathrm{B}$ atoms with trigonal-planar surrounding.

Besides octahedra, $\mathrm{B}_{12}$ icosahedra form the main building blocks of many boron-rich borides and are the dominant structural element of all allotropes of B. ${ }^{1}$ As ref. 31 and references therein show, chemical shifts of numerous icosahedral closo-(hetero)dodecaboranes have been known for more than five decades. Moreover, theoretical and experimental studies on nuclear quadrupole coupling constants of icosahedral carboranes are also reported. ${ }^{32}$ On the other hand, to the best of our knowledge there exist to date just small sets of calculated ${ }^{26,27}$ or measured $^{33-35}$ NMR data for only a few boron-rich borides with icosahedral structure elements, this is particularly true considering nuclear magnetic ${ }^{11} \mathrm{~B}$ shifts. ${ }^{19}$ Certainly there is not only the already mentioned problem of phase-pure synthesis but also the difficulty of processing measured signals. Several symmetryindependent positions of atoms may lead to complex NMR spectra, which cannot be easily understood despite the knowledge of structure investigated. The main reasons for this are that a simple correlation between the tensor components of the chemical shift or EFG with the electron density a priori is not possible and/or even no comparable values of similar compounds exist. So the common procedure in NMR spectroscopy to compare the chemical shifts of a specific atom to a closely related model system, for example $\mathrm{BO}_{4}$ tetrahedra or trigonal-planar $\mathrm{BO}_{3}$ units in borates, is not possible.

Besides, previous work $^{36}$ have tried to address isotropic chemical ${ }^{11} \mathrm{~B}$ shifts in homonuclear bonds of diboron compounds in the theoretical framework of density functional theory (DFT) applying the gauge-including projector-augmented wave (GIPAW) approach. ${ }^{37,38}$ However, there the lack of applying an appropriate reference led to computational errors of more than $14 \mathrm{ppm}$ which we think is not suitable for understanding SSNMR spectra where more particular sites may cause signal overlaps. In contrast, a recent report $^{39}$ presented evidence for calculating isotropic chemical ${ }^{11} \mathrm{~B}$ shifts of various closo-(hetero)dodecaboranes with GIPAW demonstrating an accuracy of 1.0 to $2.1 \mathrm{ppm}$ compared to experimental data depending on the applied method of reference. Furthermore, in our previous study ${ }^{40}$ on rhombohedral phases we showed that calculated chemical shifts and quadrupolar coupling constants are in excellent agreement with MAS NMR measurements (in range of $\pm 1 \mathrm{ppm})$ of the boron modification $\alpha-\mathrm{B}\left(\alpha-\mathrm{rB}_{12}\right)$ and $\mathrm{B}_{12} \mathrm{P}_{2}$ as well as other work on boron-rich borides and also molecular compounds with icosahedral structure element. There, the analysis of the EFG revealed that the highly symmetric arrangement of B atoms to icosahedra and the strength of the outericosahedral bonds lead to a mapping of the predominant bonding situation in values of the quadrupole coupling constant/ frequency.

The present study is an extension of our computational DFT investigation on NMR parameters of boron modifications and boron-rich borides which expands the available data sets of this solid state compound class. Our GIPAW calculations are complemented by measured MAS NMR spectra of the orthorhombic phases of $\mathrm{MgB}_{7}{ }^{41}$ and $\mathrm{o}-\mathrm{MgB}_{12} \mathrm{C}_{2}{ }^{42}$ which have been 
synthesized and characterized by X-ray diffraction (XRD) analysis in our laboratories. Both compounds exhibit high structural similarity in interconnected $B_{12}$ icosahedra with the inclusion of interstitial $\mathrm{B}_{2}$ or $\mathrm{C}_{2}$ dumbbell units, resulting in four $\left(\mathrm{o}-\mathrm{MgB}_{12} \mathrm{C}_{2}\right)$ or five $\left(\mathrm{MgB}_{7}\right)$ distinguishable $\mathrm{B}$ sites. Adding reasonable values for signal intensities and linewidths under guidance of the eye leads to convincing results for spectra simulation whereas the conventional fitting procedures fail to yield unambiguous parameters sets or even do not converge at all. This makes us confident to also present calculated values of the closely related $\mathrm{B}$ modification $\gamma-\mathrm{B}_{28}$. A comparison of frequently used construction schemes for pseudopotentials (PPs) shows very good agreement.

Additionally, we discuss the electronic structure applying Bader's quatum theory of atoms in molecules (QTAIM) ${ }^{43}$ in order to gain a deeper understanding in the common and different bonding features. We observe a linear correlation between the large main component $V_{z z}$ of the EFG for icosahedral atoms in outericosahedral B-B bonds with properties of the bond critical points (BCPs) from Bader's analysis. Consequently, there is a direct mapping between the electronic properties of the bonds external from the $\mathrm{B}_{12}$ unit and measureable quadrupole interaction constants, which in our opinion arises from the particular geometry of the icosahedron.

\section{Methods}

\subsection{Computational details}

Table 1 provides an overview of the crystallographic parameters of the investigated compounds. For all calculations DFT is applied using the program package Quantum ESPRESSO $(\mathrm{QE})^{44,45}$ and the generalised gradient approximation according to Perdew-Burke-Ernzerhof $(\mathrm{PBE})^{46}$ with a kinetic energy cutoff of 80 Ry for the plane-wave basis set expansion. By aid of the program CIF2CELL ${ }^{47}$ the atomic positions were generated for the corresponding primitive unit cells (see ESI $\dagger$ for details) on which we performed all calculations. We sampled the $k$-grid by the approach of Monkhorst and $\mathrm{Pack}^{48}$ with a precision smaller than $0.04 \frac{2 \pi}{\AA}$, that is for $\mathrm{MgB}_{7} 4 \times 6 \times 7$, for $\mathrm{o}-\mathrm{MgB}_{12} \mathrm{C}_{2} 6 \times 4 \times 7$, and for $\gamma-B_{28} 7 \times 6 \times 5$. Structure optimization was carried out under maintenance of the lattice constants with PPs of the Goedecker-Hartwigsen-Hutter contraction ${ }^{49}$ and with assumed

Table 1 Details of crystallographic data for the investigated compounds

\begin{tabular}{llll}
\hline & $\mathrm{MgB}_{7}{ }^{41}$ & $\mathrm{o}-\mathrm{MgB}_{12} \mathrm{C}_{2}{ }^{42}$ & $\gamma-\mathrm{B}_{28}{ }^{10}$ \\
\hline $\begin{array}{l}\text { Composition } \\
\text { Crystal system }\end{array}$ & $\begin{array}{l}\mathrm{Mg}_{4} \mathrm{~B}_{28} \\
\text { Orthorhombic }\end{array}$ & $\mathrm{Mg}_{2} \mathrm{~B}_{24} \mathrm{C}_{4}$ & $\mathrm{~B}_{28}$ \\
Space group & Imma (no. 74) & & Pnnm (no. 58) \\
& & & \\
Lattice parameters & & & \\
$a(\AA)$ & $10.4782(16)$ & $5.6133(10)$ & $5.0576(4)$ \\
$b(\AA)$ & $5.9769(9)$ & $9.828(2)$ & $5.6245(8)$ \\
$c(\AA)$ & $8.1245(19)$ & $7.9329(15)$ & $6.9884(10)$ \\
Volume $\left(\AA^{3}\right)$ & 508.8 & 437.6 & 198.8 \\
${ }^{a}$ Primitive cell. & & &
\end{tabular}

convergency for all components of all forces smaller than $2.5 \mathrm{meV}^{-1}$.

Using the GIPAW ${ }^{37,38}$ algorithm the NMR parameters were determined with PPs from normconserving construction according to Troullier Martins (NC) ${ }^{50}$ as well as with the all-electron projector augmented-wave (PAW) contraction following Kresse and Joubert. ${ }^{51}$ With these calculations the principal components of the magnetic shielding tensor are obtained as $\sigma_{x x}, \sigma_{y y}$ and $\sigma_{z z}$. The isotropic chemical shift $\delta_{\text {iso }}$ is computed by the relation $\delta_{\text {iso }}=m \cdot \sigma_{\text {iso }}+\sigma_{\text {iso }}^{\text {ref }}$ with the isotropic shielding $\sigma_{\text {iso }}=$ $\left(\sigma_{x x}+\sigma_{y y}+\sigma_{z z}\right) / 3$ and $\sigma_{\text {iso }}^{\text {ref }}$ as the isotropic shielding of a selected reference. For the reference approach we employed the two methods described in literature. ${ }^{20}$ These are on the one hand the linear regression with $m=-0.866$ and $\sigma_{\text {iso }}^{\text {ref }}=80.426 \mathrm{ppm},{ }^{39}$ and on the other hand the conservative shielding to shift conversion setting $m=-1$ and adjusting $\sigma_{\text {iso }}^{\text {ref }}$ to ensure the calculated shielding $\sigma_{\text {iso }}$ of $\left(\mathrm{B}_{12} \mathrm{H}_{12}\right)^{2-}$ leads to $\delta_{\text {iso }}$ which is measured as $-15.3 \mathrm{ppm}$ relative to boron trifluoride diethyl etherate $\mathrm{BF}_{3} \cdot \mathrm{OEt}_{2} \cdot{ }^{31}$ Following the recommendation of ref. 52 we refer to the SIMPSON/Haeberlen convention..$^{53}$ with the chemical shift anisotropy $\delta_{\text {csa }}=m \cdot\left(\sigma_{z z}-\sigma_{\text {iso }}\right)$ as well as the asymmetry $\eta_{\mathrm{cs}}=\left(\sigma_{y y}-\sigma_{x x}\right) /\left(\sigma_{z z}-\sigma_{\text {iso }}\right)$. We report the principal components of the EFG $V_{i i}$ with $\left|V_{z z}\right| \geq\left|V_{y y}\right| \geq\left|V_{x x}\right|$ as the quadrupolar coupling constant $C_{\mathrm{q}}=e Q V_{z z} / h$ and the quadrupolar asymmetry parameter $\eta_{\mathrm{q}}=\left(V_{x x}-V_{y y}\right) / V_{z z}$ using the quadrupole moment $Q\left({ }^{11} \mathrm{~B}\right)=4.059 \times 10^{-30} \mathrm{~m}^{2} .{ }^{54}$

The analysis according to Bader's QTAIM ${ }^{43}$ was accomplished with the program CRITIC2. ${ }^{55,56}$ For this purpose, the electron density calculated with PAW was mapped onto a real lattice with a three times higher refinement than chosen by QE's default settings. The convergence with respect to the applied $k$-mesh and the plane-wave cutoff was verified for all reported quantities.

The simulations of the MAS NMR spectra are obtained by applying the DMfit program ${ }^{57}$ in combination with the calculated parameters. In particular, we used the model "Quad 1st" with intensities and linewidths considering our measurements and under guidance of the eye (see ESI $\dagger$ for further information). All illustrations regarding crystal structures were created using VESTA. $^{58}$

\subsection{NMR spectroscopy}

Reported SSNMR experiments were performed on a Bruker Avance $500 \mathrm{MHz}$ solid-state NMR spectrometer (11.7 T) operating at a Larmor frequency $\nu_{1}=160.48 \mathrm{MHz}$ for ${ }^{11} \mathrm{~B}$ using $2.5 \mathrm{~mm}$ rotors. Radio frequency pulses were applied at a transverse $B_{1}$ field of Grindeq issue $125 \mathrm{kHz}$ corresponding to a $\pi / 2$ pulse width of $2 \mu \mathrm{s}$. All spectra were recorded under MAS conditions at $30 \mathrm{kHz}$ with bearing gas at ambient temperature, leading to sample temperatures of approximately $323 \mathrm{~K}$ due to frictional heating. ${ }^{11} \mathrm{~B}$ spectra were recorded using a rotor-synchronized Hahn-Echo experiment. The background signal originating from the BN-stator was recorded in a separate experiment and subtracted. According to IUPAC recommendations the spectra were referenced for the unified chemical shift scale using the residual

$\ddagger$ This implies $\left|\sigma_{z z}-\sigma_{\text {iso }}\right| \geq\left|\sigma_{x x}-\sigma_{\text {iso }}\right| \geq\left|\sigma_{y y}-\sigma_{\text {iso }}\right|$. 
protons in $\mathrm{D}_{2} \mathrm{O}$ as secondary standard and the substitution method without reshimming the magnet $\left(\Xi\left({ }^{11} \mathrm{~B}\right)=32.083974 \%\right) .{ }^{52}$

\section{Results and discussion}

\subsection{Structural aspects, bonding situation and electronic structure}

All investigated compounds crystallize in the orthorhombic crystal system and are closely related to each other regarding their structure. The main structural element are icosahedra of $\mathrm{B}$ atoms which are connected to interstitial dumbbell units. In this work we denote the sites of the $\mathrm{B}_{12}$ unit from $\mathrm{B}(1)$ to $\mathrm{B}(4)$. They form endohedral bonds within the icosahedron and exohedral bonds to the surrounding. The interstitial sites in $\mathrm{MgB}_{7}$ and $\gamma-\mathrm{B}_{28}$ are labeled with $\mathrm{B}(5)$. With the nomenclature introduced in this work we aim to map the structural units as convenient as possible with regard to the exohedral bonding situation. The common structural arrangement of the compounds can be understood by the example of $\mathrm{MgB}_{7}$ shown in Fig. 1. In addition, Fig. 1c provides an overview of the used labeling of atoms.

Via the $\mathrm{B}(1)$ position, the $\mathrm{B}_{12}$ units are linked to rods along the a axis and form a primitive hexagonal packing. Perpendicular to the rods, the icosahedra are connected to each other in layers within the $b c$-plane over the $\mathrm{B}(2)$ atoms. The $\mathrm{Mg}(2)$ atoms alternate with dumbbells of $\mathrm{B}(5)$ atoms in the trigonally prismatic voids of the hexagonal primitive rod packing, whereas the $\mathrm{Mg}(1)$ atoms sit on two of the side faces of the prisms. The interstitial unit is coordinated by six icosahedra via four $\mathrm{B}(3)$ and two $\mathrm{B}(4)$ positions. In all compounds under investigation, the atoms of the dumbbell,

(b)

(a)
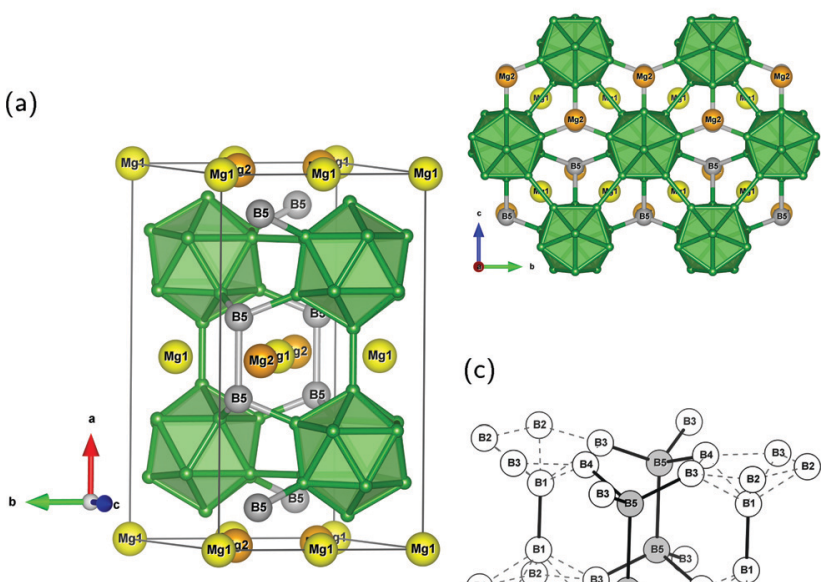

(c)

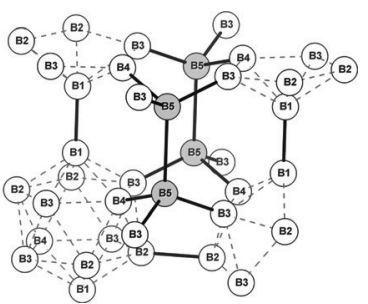

Fig. 1 Crystal structure of $\mathrm{MgB}_{7}$ and numbering system of the different $\mathrm{B}$ sites used in this work; both $\mathrm{Mg}$ sites and the interstitial $\mathrm{B}_{2}$ dumbbell are colored for the sake of appearance: (a) orthorhombic unit cell; (b) projection in [100] direction; (c) numbering of distinguishable B atoms used throughout in this work with exohedral (full) and endohedral (dashed) connections. the $\mathrm{B}(1)$ and $\mathrm{B}(4)$ of the same icosahedron are located in a mirror plane $\sigma_{\mathrm{h}}$. And since a $C_{2}$ axis runs through the centers of the innericosahedral $\mathrm{B}\left({ }_{3}\right)-\mathrm{B}\left({ }_{3}\right)$ connections, the center of mass of the $\mathrm{B}_{12}$ unit is also a center of inversion. Thus, the polyhedron's point group reduces from $I_{\mathrm{h}}$ to $C_{2 \mathrm{~h}}$.

According to Wade, ${ }^{4}$ the formation of an icosahedral $\mathrm{B}_{12}$ skeleton requires 26 electrons for the $n+1$ bonding orbitals. However, in order for each atom to give rise to an exohedral 2-electron-2-center $(2 \mathrm{e} 2 \mathrm{c})$ bond, $2 e$ are needed in addition to the valency of $12 \times 3 e=36 e$. In case of $\mathrm{MgB}_{7}\left(\mathrm{Mg}_{2} \mathrm{~B}_{12} \mathrm{~B}_{2}\right)$, this is accomplished by the formal transfer of overall $4 e$ by two $\mathrm{Mg}$ atoms to the $\mathrm{B}_{12}$ and the $\mathrm{B}_{2}$ unit. For $\mathrm{o}-\mathrm{MgB}_{12} \mathrm{C}_{2}$ the interstitial $\mathrm{C}$ atoms contribute $4 e$ each to their four bonds and the $\operatorname{Mg}(1)$ site provides its additional $2 e$ to the icosahedron. Therefore, these two compounds are also called electron-precise as there are exclusively exohedral $2 \mathrm{e} 2 \mathrm{c}$ bonds and confirmed by color (red $\mathrm{MgB}_{7}$; colorless $\mathrm{o}-\mathrm{MgB}_{12} \mathrm{C}_{2}$ ), UV/VIS spectra and bandgap calculations. $^{7,41,42}$

The atomic distances are listed in Table 2. In $\mathrm{MgB}_{7}$ and $\mathrm{o}-\mathrm{MgB}_{12} \mathrm{C}_{2}$, the intericosahedral $\mathrm{B}(1)-\mathrm{B}(1)$ and $\mathrm{B}(2)-\mathrm{B}(2)$ distances are below the respective average values for the innericosahedral $\mathrm{B}-\mathrm{B}$ distances. Furthermore, these $\mathrm{B}(1)-\mathrm{B}(1)$ lengths are the shortest in all compounds. Within the $\mathrm{B}_{12}$ units, the atomic distances differ only slightly from the mean values, indicating minor deviations from $I_{\mathrm{h}}$ symmetry. However, notable differences between $\mathrm{MgB}_{7}$ and $\mathrm{o}-\mathrm{MgB}_{12} \mathrm{C}_{2}$ occur for distances related to the interstitial units. The lengths between the icosahedra and the $\mathrm{C}_{2}$ dumbbell are smaller and the atomic separation of $2.308 \AA$ within the $\mathrm{B}_{2}$ unit is significantly larger than $1.726 \AA$ for $\mathrm{C}-\mathrm{C}$.

Despite the high structural similarity to $\mathrm{MgB}_{7}$ and $\mathrm{o}-\mathrm{MgB}_{12} \mathrm{C}_{2}$, the exohedral bonding situation between the $\mathrm{B}_{12}$ icosahedron and the interstitial $B_{2}$ dumbbell in $\gamma-B_{28}$ turns out to be very

Table 2 Selected distances of $\mathrm{MgB}_{7}, \mathrm{O}-\mathrm{MgB}_{12} \mathrm{C}_{2}$ and $\gamma-\mathrm{B}_{28}$ in $\AA$ according to optimized atom positions

\begin{tabular}{|c|c|c|c|}
\hline & $\mathrm{MgB}_{7}(\mathrm{X}=\mathrm{B}(5))$ & $\mathrm{o}-\mathrm{MgB}_{12} \mathrm{C}_{2}(\mathrm{X}=\mathrm{C})$ & $\gamma-\mathrm{B}_{28}(\mathrm{X}=\mathrm{B}(5))$ \\
\hline \multicolumn{4}{|c|}{ Intraicosahedral } \\
\hline $\mathrm{B}(1)-\mathrm{B}(2)$ & 1.815 & 1.755 & 1.782 \\
\hline $\mathrm{B}(1)-\mathrm{B}(3)$ & 1.830 & 1.767 & 1.787 \\
\hline $\mathrm{B}(1)-\mathrm{B}(4)$ & 1.852 & 1.759 & 1.758 \\
\hline $\mathrm{B}(2)-\mathrm{B}(2)$ & 1.861 & 1.813 & 1.750 \\
\hline $\mathrm{B}(2)-\mathrm{B}(3)$ & 1.781 & 1.787 & 1.849 \\
\hline $\mathrm{B}(2)-\mathrm{B}^{\prime}(3)^{a}$ & 1.848 & 1.785 & 1.833 \\
\hline $\mathrm{B}(2)-\mathrm{B}(4)$ & 1.791 & 1.800 & 1.813 \\
\hline $\mathrm{B}(3)-\mathrm{B}(3)$ & 1.784 & 1.864 & 1.868 \\
\hline $\mathrm{B}(3)-\mathrm{B}(4)$ & 1.820 & 1.789 & 1.764 \\
\hline Average & 1.818 & 1.787 & 1.802 \\
\hline \multicolumn{4}{|c|}{ Intericosahedral } \\
\hline $\mathrm{B}(1)-\mathrm{B}(1)$ & 1.757 & 1.632 & 1.664 \\
\hline $\mathrm{B}(2)-\mathrm{B}(2)$ & 1.806 & 1.734 & \\
\hline $\mathrm{B}(2)-\mathrm{B}(3)$ & & & 1.834 \\
\hline \multicolumn{4}{|l|}{ Others } \\
\hline$B(2)-X$ & 2.937 & 2.922 & 1.915 \\
\hline$B(3)-X$ & 1.747 & 1.660 & 2.085 \\
\hline$B(4)-X$ & 1.795 & 1.647 & 1.674 \\
\hline $\mathrm{X}-\mathrm{X}$ & 2.308 & 1.726 & 1.729 \\
\hline
\end{tabular}


versatile (see Fig. 2). With its structure determination,, ${ }^{9,10}$ especially the interactions between the $\mathrm{B}(2)$ and $\mathrm{B}(3)$ positions with $\mathrm{B}(5)$ have been intensively discussed..$^{10-16}$ One reason are the atomic distances $\mathrm{B}(2)-\mathrm{B}(3), \mathrm{B}(2)-\mathrm{B}(5)$ and $\mathrm{B}(3)-\mathrm{B}(5)$ outside the polyhedron shown in Table 2, which are in the range of endohedral bonds of all listed compounds. This may cause the introduced labeling of $\mathrm{B}(2)$ and $\mathrm{B}(3)$ in Fig. 1c to seem less clear. Note that we refer to $\mathrm{B}(2)$ as the position where the shortest innericosahedral connection line $\mathrm{B}(2)-\mathrm{B}(2)$ is perpendicular to $\sigma_{\mathrm{h}}$. The other main reason for the debate on the bonding situation in $\gamma-\mathrm{B}_{28}$ stems by results from QTAIM analysis of the charge distribution, which we discuss in detail below.

From a formal point of view, there is no electron donor in $\gamma-\mathrm{B}_{28}$ (like $\mathrm{Mg}$ in $\mathrm{MgB}_{7}$ and $\mathrm{o}-\mathrm{MgB}_{12} \mathrm{C}_{2}$ ) and consequently there are only $36 e-26 e=10 e$ available to the exohedral interactions and so not all of these can be $2 \mathrm{e} 2 \mathrm{c}$ bonds. As our results agree well with those of Mondal et al. ${ }^{13}$ we follow their interpretation. For the exohedral $2 \mathrm{e} 2 \mathrm{c}$ bonds between the $\mathrm{B}_{12}$ units via the $\mathrm{B}(1)$ atoms, a total of $2 e$ are required. Between the $\mathrm{B}(4)$ and $\mathrm{B}(5)$ atoms there are also $2 \mathrm{e} 2 \mathrm{c}$ bonds for which $2 e$ are needed. The $\mathrm{B}(2)$ atoms of an icosahedron form polar-covalent 2-electron-3center $(2 \mathrm{e} 3 \mathrm{c})$ bonds with a $\mathrm{B}(5)$ atom of the dumbbell (see Fig. $2 \mathrm{~b}$ ) and contribute overall $4 \times 1 / 2 e=2 e$ per icosahedron. Finally, the exohedral $\mathrm{B}(2)-\mathrm{B}(3)$ bonds are 1-electron-2-center $(1 \mathrm{e} 2 \mathrm{c})$ bonds for which an icosahedron may provide its remaining $4 \times 1 / 2 e=2 e$. Overall one $\mathrm{B}_{12}$ polyhedron in $\gamma-\mathrm{B}_{28}$ constitutes eight $1 \mathrm{e} 2 \mathrm{c}$, four $2 \mathrm{e} 2 \mathrm{c}$ and two $2 \mathrm{e} 3 \mathrm{c}$ exohedral bonds (see also Fig. 2a).

Table 3 gives an overview of the results from QTAIM analysis. In this topological partitioning scheme, the electron density is split into basins fulfilling the zero flux condition along their boundary surface. Each region is assigned to a maximum within interpreted as atom. The respective charges can be derived from the summed charge density within the atomic basins. In general these indicate charge shifts from the electropositive to the electronegative element. The BCPs correspond to the minima on bonding paths between two atoms, while ring critical points (RCPs) are defined by closed paths of bonded atoms. Type and (a)

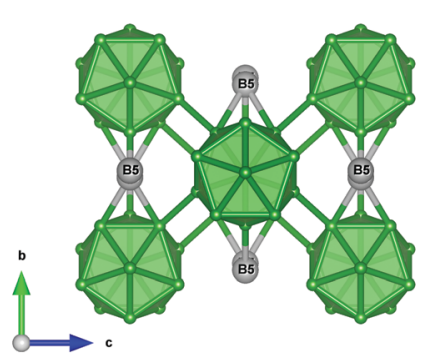

(b)

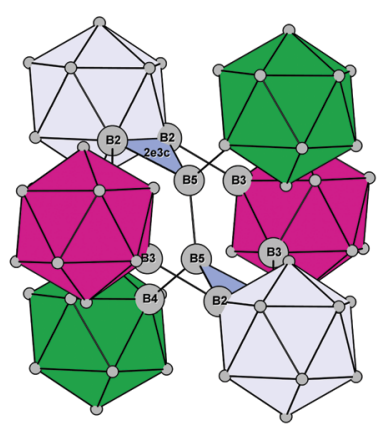

(a)

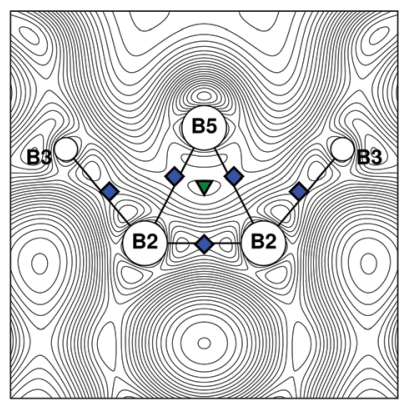

(b)

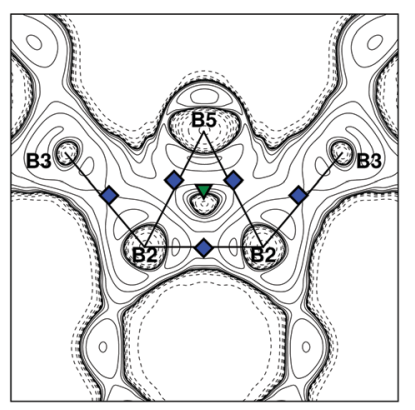

Fig. 3 Properties of calculated electron charge density (PAW) with BCPs (diamond) and RCP (triangle) in $\mathrm{B}(2)-\mathrm{B}(2)-\mathrm{B}(5)$ plane of $\gamma-\mathrm{B}_{28}$ (see also Fig. 2): (a) contour lines of density from 0.05 to 2.0 e $\AA^{-3}$. (b) Positive (solid) and negative (dashed) contour lines of Laplacian for $\pm(2,4,8) \times 10^{n}$ e $\AA^{-5}$ $(-3 \leq n \leq 3)$.

strength of the bonding interaction correlates with values of density and Laplacian at the corresponding points.

The properties of the calculated charge distributions in Table 3 are consistent with results of the (linear)-augmentedplane-waves (LAPW) method ${ }^{7}$ and are also in agreement with ref. 9 and 13 for $\gamma-\mathrm{B}_{28}$. Listed values of the BCPs are associated with the exohedral bond. The negative Laplacians indicate a dominant covalent bonding character. With the exception of $\mathrm{B}(2)-\mathrm{B}(5)$ in $\gamma-\mathrm{B}_{28}$, the ellipticities on all shown BCPs (not shown) are close to zero, meaning that all other exohedral interactions can be assumed as straight $\sigma$ bonds. ${ }^{59}$

Table 3 Parameters from Bader's QTAIM analysis from PAW calculations of the optimized structures with charge $q_{\text {PAW }}$ in $e$ and properties of the $\mathrm{BCP}$ at $r_{\mathrm{BCP}}$ with charge density $\rho$ in $e \AA^{-3}$, the Laplacian $\nabla^{2} \rho$ in $e \AA^{-5}$ and $d_{\mathrm{BCP}}$ as distance between atoms and the BCP in $\AA$

\begin{tabular}{|c|c|c|c|c|c|c|}
\hline \multirow[b]{2}{*}{ Compound } & \multirow[b]{2}{*}{ Site } & \multicolumn{2}{|c|}{ QTAIM charge } & \multicolumn{3}{|c|}{ Property of BCP } \\
\hline & & Ref. $7^{b}$ & $q_{\mathrm{PAW}}$ & $\rho\left(r_{\mathrm{BCP}}\right)$ & $\nabla^{2} \rho\left(r_{\mathrm{BCP}}\right)$ & $d_{\mathrm{X}-\mathrm{BCP}}, d_{\mathrm{BCP}-\mathrm{Y}}$ \\
\hline \multirow[t]{7}{*}{$\mathrm{MgB}_{7}$} & $\mathrm{~B}(1)$ & -0.10 & -0.07 & 0.924 & -6.713 & $0.879,0.879$ \\
\hline & $\mathrm{B}(2)$ & -0.20 & -0.22 & 0.817 & -4.155 & $0.905,0.905$ \\
\hline & $\mathrm{B}(3)$ & -0.13 & -0.14 & 0.905 & -6.325 & $0.917,0.831$ \\
\hline & $\mathrm{B}(4)$ & -0.30 & -0.35 & 0.832 & -5.286 & $0.951,0.849$ \\
\hline & $\mathrm{B}(5)$ & -0.56 & -0.53 & 0.420 & -0.434 & $1.155,1.155$ \\
\hline & $\operatorname{Mg}(1)$ & 1.63 & 1.57 & & & \\
\hline & $\operatorname{Mg}(2)$ & 1.66 & 1.68 & & & \\
\hline \multirow[t]{6}{*}{$\mathrm{o}-\mathrm{MgB}_{12} \mathrm{C}_{2}$} & $\mathrm{~B}(1)$ & -0.10 & -0.07 & 1.123 & -9.646 & $0.816,0.816$ \\
\hline & $\mathrm{B}(2)$ & -0.31 & -0.34 & 0.894 & -5.814 & $0.869,0.869$ \\
\hline & $\mathrm{B}(3)$ & 0.46 & 0.48 & 1.051 & -7.888 & $0.571,1.090$ \\
\hline & $\mathrm{B}(4)$ & 0.55 & 0.57 & 1.062 & -4.420 & $0.557,1.090$ \\
\hline & $\mathrm{C}$ & -1.58 & -1.63 & & & \\
\hline & $\mathrm{Mg}$ & 1.68 & 1.71 & & & \\
\hline \multirow[t]{5}{*}{$\gamma-\mathrm{B}_{28}$} & $\mathrm{~B}(1)$ & 0.06 & 0.05 & 1.091 & -9.725 & $0.832,0.832$ \\
\hline & $\mathrm{B}(2)$ & -0.17 & -0.19 & $0.554^{c d}$ & $-1.137^{c d}$ & $1.077,0.842^{c}$ \\
\hline & $\mathrm{B}(3)$ & 0.00 & 0.01 & $0.758^{e}$ & $-4.432^{e}$ & $0.908,0.936^{e}$ \\
\hline & $\mathrm{B}(4)$ & 0.04 & 0.07 & 1.089 & -9.835 & $0.829,0.847$ \\
\hline & $\mathrm{B}(5)$ & 0.24 & 0.26 & 0.980 & -8.242 & $0.865,0.865$ \\
\hline
\end{tabular}

${ }^{a}$ Between exohedral bonds or B(5)-B(5). ${ }^{b}$ LAPW (PW91-LDA) without geometry optimization. ${ }^{c}$ Between $\mathrm{B}(2)-\mathrm{B}(5)$ (see Fig. 3). ${ }^{d}$ RCP of $\mathrm{B}(2)-$ $\mathrm{B}(2)-\mathrm{B}(5): \rho\left(r_{\mathrm{RCP}}\right)=0.538 e \AA^{-3}, \nabla^{2} \rho\left(r_{\mathrm{RCP}}\right)=-0.103 e \AA^{-5}$ (see Fig. 3 ). ${ }^{e}$ Between $\mathrm{B}(2)-\mathrm{B}(3)$ (see Fig. 3). 
In the compounds containing $\mathrm{Mg}$, the metal transfers electrons to the covalent non-metal framework. However, the charges listed above deviate from electron counting rule according to Wade, which assumes equal distributed bonding fractions. As can be seen in case of $\mathrm{o}-\mathrm{MgB}_{12} \mathrm{C}_{2}$, the BCPs are shifted towards $\mathrm{B}$ due to the more electronegative character of the $\mathrm{C}_{2}$ dumbbell. This leads to a reduction of the assigned atomic basins and, therefore, to a significant difference in charges of $\mathrm{B}(3)$ and $\mathrm{B}(4)$ comparing $\mathrm{MgB}_{7}$ with $\mathrm{o}-\mathrm{MgB}_{12} \mathrm{C}_{2}$.

The given $\mathrm{BCPs}$ from $\mathrm{B}(1)$ to $\mathrm{B}(4)$ of $\mathrm{MgB}_{7}$ and $\mathrm{o}-\mathrm{MgB}_{12} \mathrm{C}_{2}$ relate to the strongest bond formation to be exohedral due to highest values of electron density. For $\gamma-B_{28}$, this result is only true for the $\mathrm{B}(1)$ and $\mathrm{B}(4)$ sites, whereas $\mathrm{B}(2)$ and $\mathrm{B}(3)$ show lower densities than their endohedral bonds. The reason for this is the special bonding situation of these two atomic positions. As shown in Fig. 2, the calculated electron density exhibits exohedral BCPs between $\mathrm{B}(2)$ and $\mathrm{B}(3)$ and between $\mathrm{B}(2)$ and $\mathrm{B}(5)$. Contrary to the $\mathrm{B}(2)-\mathrm{B}(3)-\mathrm{B}(5)$ multi-center bond postulated by Rulis et al., ${ }^{16}$ we find an RCP only within the $\mathrm{B}(2)-$ $\mathrm{B}(2)-\mathrm{B}(5)$ formation. The ellipticity of the $\mathrm{BCP}$ on $\mathrm{B}(2)-\mathrm{B}(5)$ (see also Fig. 2) is with 3.6 in typical order of magnitude for endohedral 2e3c icosahedral bonds in molecules ${ }^{5}$ and solid phases ${ }^{7}$ but also for the exohedral equatorial bond in $\alpha-\mathrm{rB}_{12}{ }^{60}$

Our results, in particular the values $\rho\left(r_{\mathrm{BCP}}\right)$ and $\rho\left(r_{\mathrm{RCP}}\right)$ of the points illustrated in Fig. 2 are in excellent agreement with the analysis of the measured electron density of $\gamma-\mathrm{B}_{28} \cdot{ }^{13}$ Mondal et $a{ }^{13}{ }^{13}$ conclude from the long bond distance $(\approx 1.83 \AA)$ in combination with the small $\rho\left(r_{\mathrm{BCP}}\right)$ that $\mathrm{B}(2)-\mathrm{B}(3)$ is a $1 \mathrm{e} 2 \mathrm{c}$ bond. Furthermore, the $\mathrm{B}(2)-\mathrm{B}(2)-\mathrm{B}(5) \mathrm{RCP}$ indicates a $2 \mathrm{e} 3 \mathrm{c}$ bond, with the BCPs along $\mathrm{B}(2)-\mathrm{B}(5)$ being noticeable shifted towards $\mathrm{B}(5)$, and thus should be considered a polar-covalent bond (compare also Fig. 3 of ref. 13 with Fig. 2). As a recent experimental and theoretical investigation of the mechanical properties suggests, this bond formation to the interstitial $B_{2}$ unit are called "ultrasoft bonds". ${ }^{17}$

Despite the same structural element of a $B_{2}$ dumbbell in $\mathrm{MgB}_{7}$ and $\gamma-\mathrm{B}_{28}$, we observe different electronic situations within the interstitial units. In $\mathrm{MgB}_{7}$ the $\mathrm{BCPs}$ of $\mathrm{B}(3)-\mathrm{B}(5)$ and $\mathrm{B}(4)-\mathrm{B}(5)$ are shifted to $\mathrm{B}(5)$, which corresponds to the picture of an electropositive bonding partner. And indeed, Bader charge is accumulated by the icosahedron $\left[\mathrm{Mg}_{2}^{3.25+} \mathrm{B}_{12}^{2.19-} \mathrm{B}_{2}^{1.06-}\right]$ as one would expect according to Wade. In $\gamma-\mathrm{B}_{28}$, on the other hand, the $\mathrm{B}_{2}$ unit is positively charged $\left[\mathrm{B}_{2}^{0.52+}\right]$. Again, the BCPs of the $\mathrm{B}(2)-\mathrm{B}(5)$ bonds are shifted towards the interstitial unit and the icosahedron accumulates negative charge density. This overall behavior is consistent with the conclusion of Häussermann and Mikhaylushkin ${ }^{11}$ that the peculiar $B_{2}$ dumbbell acts as an electron reservoir for the $\mathrm{B}_{12}$ polyhedron. There is a significant excess of electrons in $\mathrm{MgB}_{7}$ which is transferred to $\mathrm{B}_{2}$. This accumulation of charge leads to an increased bond distance of $\mathrm{B}(5)-\mathrm{B}(5)$ with low electron density at the BCP as well as small $\nabla^{2} \rho$ and finally to a weak covalent interaction. In contrast to that, electron density of the interstitial unit is subtracted by the icosahedra in $\gamma-\mathrm{B}_{28}$. Accordingly, the $\mathrm{B}_{2}$ dumbbell forms a strong covalent bond with a typical distance of $1.729 \AA$ (comparable to $\mathrm{C}-\mathrm{C}$ in $\mathrm{o}-\mathrm{MgB}_{12} \mathrm{C}_{2}$ ). Yet referring to Mondal et al. ${ }^{13}$ this represents no explicit charge transfer between icosahedron and interstitial unit. Based on the measured electron density, they find that $1 / 3 e$ of $\mathrm{B}(5)$ is given into the $2 \mathrm{e} 3 \mathrm{c}$ bond, so that $1 / 6 e$ is available for each $\mathrm{B}(2)$ atom.

The density of states (DOSs) presented in Fig. 4 are in very good agreement with other work for $\mathrm{MgB}_{7},{ }^{7,8,41} \mathrm{o}-\mathrm{MgB}_{12} \mathrm{C}_{2}{ }^{6,7}$ and $\gamma-\mathrm{B}_{28} \cdot{ }^{10,14,16}$ All compounds are characterized by a significant bandgap of at least $1.5 \mathrm{eV}$. Regarding the icosahedron the atomic relative DOSs show a strong hybridization of the $\mathrm{s} / \mathrm{p}$ states and hardly any differences amongst the investigated compounds. In general, the interstitial unit influences the DOS especially in the range of -5 to 0 . But in contrast to all $\mathrm{B}(5)$ sites, the DOS of $\mathrm{o}-\mathrm{MgB}_{12} \mathrm{C}_{2}$ is dominated around $-13 \mathrm{eV}$ by the low-lying s-states of the interstitial $\mathrm{C}$ atoms. A comparison of the interstitial $\mathrm{B}(5)$ atoms in $\mathrm{MgB}_{7}$ with those in $\gamma-\mathrm{B}_{28}$ reveals a much higher population of $\mathrm{p}$-states near the Fermi level, while the population of s-states rapidly decreases between $-4 \mathrm{eV}$ and $-3 \mathrm{eV}$ in $\mathrm{MgB}_{7}$ in analogy to the one of $\mathrm{C}$ in $\mathrm{o}-\mathrm{MgB}_{12} \mathrm{C}_{2}$. Overall, in $\gamma-\mathrm{B}_{28}$ the s-states are more diffuse and show a more distinct interaction to p-states in the aforementioned region. In this respect, $\mathrm{B}(5)$ in $\mathrm{MgB}_{7}$ and $\mathrm{C}$ in $\mathrm{o}-\mathrm{MgB}_{12} \mathrm{C}_{2}$ show similarities that are evidently extended above the Fermi level at about $2.5 \mathrm{eV}$ : Both interstitial atoms show significantly lower contribution, while $\mathrm{B}(5)$ in $\gamma-\mathrm{B}_{28}$ becomes dominant compared to an icosahedral $\mathrm{B}$ atom. This is a manifestation of the earlier described behavior of the $\mathrm{B}_{2}$ dumbbell being a reservoir or buffer for electrons. While in $\gamma-B_{28}$ further accumulation of negative charge is possible, the states of $\mathrm{B}(5)$ in $\mathrm{MgB}_{7}$ are strongly occupied comparable to the $\mathrm{sp}^{3}$ hybridized $\mathrm{C}$ in $\mathrm{o}-\mathrm{MgB}_{12} \mathrm{C}_{2}$.

\subsection{Calculated NMR parameters and measured MAS NMR spectra}

The calculated parameters of the chemical shift and quadrupole coupling are presented in Table 4 . To the best of our knowledge, this is the first time that calculated ${ }^{11} \mathrm{~B}$ shifts of boron-rich borides are reported to this extent. Since usually only the absolute value of the quadrupole coupling constant can be determined experimentally, ${ }^{19}$ we refer to $\left|C_{\mathrm{q}}\right|$. The corresponding components of the EFGs will be discussed below in further detail.

Relative to the values of conservative referencing, the results of PPs with NC and PAW constructions are in good agreement. Only for the shifts of $\mathrm{B}(5)$ in $\gamma-\mathrm{B}_{28}$, a difference of $0.6 \mathrm{ppm}$ leads to the interstitial atoms being more shielded than $\mathrm{B}(4)$ according to NC. Likewise, the $\left|C_{\mathrm{q}}\right|$ and $\eta_{\mathrm{q}}$ values show only minor dependence on the applied type of PP. The determined shifts with respect to the linear regression within the NC approach deviate from the conservative method the more the shielding of $B$ increases. The used regression parameters are mainly derived from rather highly shielded ${ }^{11} \mathrm{~B}$ species of closo-(hetero)dodecaboranes and are therefore unsuitable for deshielded nuclei, ${ }^{39}$ whereas the conservative reference to $\mathrm{B}_{12} \mathrm{H}_{12}{ }^{2-}$ is more stable for a larger shift range of chemical shifts as will become more clear considering the measured MAS NMR spectra. 
(a)

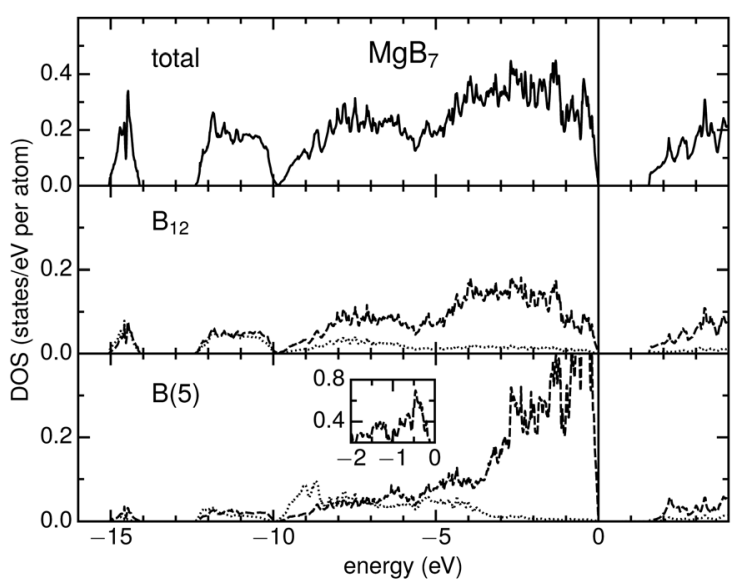

(b)

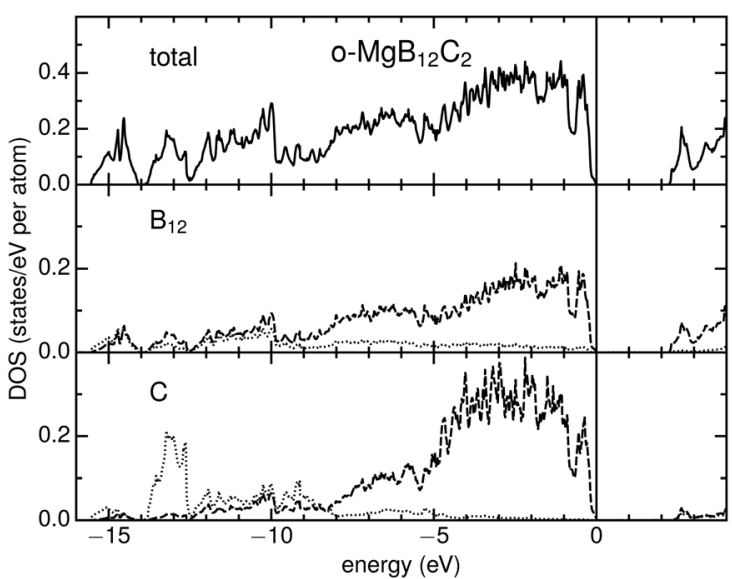

(c)



Fig. 4 Total (top) and local partial electronic density of states (center: icosahedral $B_{12}$ unit; bottom: interstitial $X_{2}$ unit) of (a) $\mathrm{MgB}_{7}$, (b) o- $\mathrm{MgB}_{12} \mathrm{C}_{2}$ and (c) $\gamma-B_{28}$ for angular components of $s$ (dotted) and $p$ (dashed).

Similar to the calculation of the shifts of the rhombohedral phases, ${ }^{40}$ the consideration of the correction by the bare macroscopic susceptibility $\chi_{\text {bare }}$ plays a crucial role, since it is about $-0.7 \times 10^{-9} \mathrm{~m}^{3} \mathrm{~kg}^{-1}$ for the investigated compounds and, thus, contributes to an overall difference of about $14 \mathrm{ppm}$ (see $\mathrm{ESI} \dagger$ for details). For comparison, it is in the same range of magnitude as of other diamagnetic boron-rich borides like $\mathrm{YB}_{6}$ and $\mathrm{YB}_{4}$ with measured values of $-2.6 \times 10^{-9} \mathrm{~m}^{3} \mathrm{~kg}^{-1}$ (ref. 61) and $-5.9 \times 10^{-9} \mathrm{~m}^{3} \mathrm{~kg}^{-1}$ (ref. 62) or $\mathrm{LaB}_{4}$ with $-1.0 \times 10^{-9} \mathrm{~m}^{3} \mathrm{~kg}^{-1}, 63$ respectively.

As the bare induced field $\mathrm{B}_{\mathrm{in}}^{(1)}(\mathbf{G}=\mathbf{0})$ is affected by the surface currents of the sample the corresponding magnetic shielding $\boldsymbol{\sigma}(\mathbf{G}=\mathbf{0})$ is not a bulk property. ${ }^{37,64,65}$ Being consistent with the on-site approximation for the reconstruction current it is computed via $\chi_{\text {bare }}$. For a general shape of nonmagnetic media with small, isotropic susceptibility, it can be shown that the shielding is related to the calculated $\sigma$ by $\sigma_{i j}^{\text {shape }} \simeq \sigma_{i j}-\delta_{i j} 4 \pi \bar{\chi}$ $\left(1-n_{j}\right)$ with $\bar{\chi}=\operatorname{tr}\left(\sigma_{\text {bare }}\right) / 3$ and $n_{k}$ as depolarization coefficient. ${ }^{66}$ Assuming a spherical sample shape $\left(n_{x}=n_{y}=n_{z}=1 / 3\right)$ we follow Mauri et al. ${ }^{64}$ that in case of MAS measurements on a solid with isotropic $\chi_{\text {bare }}, \boldsymbol{\sigma}$ is independent of the particular shape of the sample. Thus, the application and quality of this correction depends on a high degree of isotropy for $\chi_{\text {bare }}$. To evaluate this, we may describe the extent of anisotropy following the definition of fractional anisotropy $\left(\mathrm{fa}_{x}\right)$ known from diffusion processes. ${ }^{67,68} \mathrm{fa}_{\chi}$ is a scalar that quantifies the pointedness of an ellipsoid defined by the eigenvectors and eigenvalues of $\chi_{\text {bare }}$ with values between 0 (case of a sphere) and 1 (only one nonzero eigenvalue).§ With values from the PAW calculations we obtain for the here investigated compounds $\mathrm{fa}_{y}\left(\mathrm{MgB}_{7}\right)=$ $5.2 \%, \mathrm{fa}_{\gamma}\left(\mathrm{o}-\mathrm{MgB}_{12} \mathrm{C}_{2}\right)=5.1 \%$ and $\mathrm{fa}_{\gamma}\left(\gamma-\mathrm{B}_{28}\right)=3.7 \%$ comparable to those of our previous investigation ${ }^{40}$ with $\mathrm{fa}_{\chi}\left(\mathrm{B}_{12} \mathrm{P}_{2}\right)=2.1 \%$, $\mathrm{fa}_{\chi}\left(\mathrm{B}_{12} \mathrm{As}_{2}\right)=2.5 \%$ and $\mathrm{fa}_{\chi}\left(\mathrm{B}_{12} \mathrm{O}_{2}\right)=5.4 \%$ indicating a minor degree of anisotropy ${ }^{69}$ and confirming the applicability of the used correction.

With respect to the isotropic shifts, we recognize for the two very similar compounds $\mathrm{MgB}_{7}$ and $\mathrm{o}-\mathrm{MgB}_{12} \mathrm{C}_{2}$ an order of the form $\delta_{\text {iso }}(\mathrm{B}(1))<\delta_{\text {iso }}(\mathrm{B}(2))<\delta_{\text {iso }}(\mathrm{B}(3))<\delta_{\text {iso }}(\mathrm{B}(4))$. This means that although the charge distribution according to Bader is different, the chemical shifts follow a pattern with respect to the icosahedral building parts in the structures. We find a high similarity in $\gamma-\mathrm{B}_{28}$ with $\delta_{\text {iso }}(\mathrm{B}(2))$ deviating from this pattern, possibly because of the special bonding situation of $\mathrm{B}(2)-\mathrm{B}(5)$. In addition, for this compound the shift range is much smaller than in $\mathrm{MgB}_{7}$ or o- $\mathrm{MgB}_{12} \mathrm{C}_{2}$ and a general assignment of $\delta_{\text {iso }}$ to a certain $\mathrm{B}$ site is not possible. For example, the $\mathrm{B}(1)$ sites in $\mathrm{MgB}_{7}$ and $\mathrm{o}-\mathrm{MgB}_{12} \mathrm{C}_{2}$ show a difference in shifts of more than $10 \mathrm{ppm}$ and $\mathrm{B}(1)$ in $\mathrm{MgB}_{7}$ is obtained in a similar shift range as $\mathrm{B}(2)$ in o$\mathrm{MgB}_{12} \mathrm{C}_{2}$. Also, regarding the $\mathrm{B}(5)$ atoms in $\mathrm{MgB}_{7}$ and $\gamma-\mathrm{B}_{28}$ the calculated isotropic shifts differ strongly by about $40 \mathrm{ppm}$. The $\delta_{\text {iso }}$ of the borides investigated here are therefore neither correlated with the crystallographic position nor with the Bader charge. Probably the amount of overall charge separation has an influence on the broadness of the shift range for $\mathrm{MgB}_{7}$, $\mathrm{o}-\mathrm{MgB}_{12} \mathrm{C}_{2}$ and $\gamma-\mathrm{B}_{28}$. This correlation is also suggested in the rhombohedral phases, where we obtain from $\alpha-\mathrm{rB}_{12}, \mathrm{~B}_{12} \mathrm{As}_{2}$ over

$\S$ According to ref. 67 and 68 we use $\mathrm{fa}_{\chi}=\sqrt{\left(3-1 / \operatorname{tr}\left\{\boldsymbol{R}^{2}\right\}\right) / 2}$ with $\boldsymbol{R}=\chi_{\text {bare }} /$ $\operatorname{tr}\left\{\chi_{\text {bare }}\right\}$. 
Table 4 Chemical shift parameters $\delta_{\text {iso, }} \delta_{\text {csa }}$ in ppm and $\eta_{\mathrm{cs}}$ together with the quadrupolar coupling constants $\left|C_{\mathrm{q}}\right|$ and asymmetry parameter $\eta_{\mathrm{q}}$ calculated with normconserving Troullier-Martins type (NC) and projector augmented-wave (PAW) PPs; expected shift for the center of gravity of the central transition $\delta_{\mathrm{cg}}(\mathrm{ppm})$ according to PAW values and the shift from the central transition fit of the recorded spectra $\delta_{\mathrm{ctf}}( \pm 0.8 \mathrm{ppm})$



${ }^{a}$ Referencing according to ref. $39, \delta_{\mathrm{csa}}^{\mathrm{reg}} / \delta_{\mathrm{csa}}^{\mathrm{ref}}=0.866$ is assumed (see also methods of ref. 40 for details). ${ }^{b} \delta_{\mathrm{cg}}=\delta_{\text {iso }}+\delta_{\mathrm{q}}$ with $\delta_{\mathrm{q}}=-C_{\mathrm{q}}{ }^{2}\left(1+\eta_{\mathrm{q}}{ }^{2} / 3\right) /$ $\left(40 \nu_{1}\right) \cdot{ }^{19,21,22}{ }^{c}$ For details of the central transition fit see ESI.

$\mathrm{B}_{12} \mathrm{P}_{2}$ to $\mathrm{B}_{12} \mathrm{O}_{2}$ an increase in both, the charge separation and the corresponding shift range. ${ }^{40}$

The quadrupole coupling constant of $\mathrm{B}(1)$ belongs with values in the range of 1350 to $1750 \mathrm{kHz}$ to the highest values of all polyhedral atoms within a compound, while we find much smaller values for $\mathrm{B}(2)$. Especially the $\left|C_{\mathrm{q}}\right|$ of $\mathrm{B}(1)$ reported here correspond very well with those of rhombohedral phases, which connect $\mathrm{B}_{12}$ icosahedra exohedral in polar position via $2 \mathrm{e} 2 \mathrm{c}$ bonds. ${ }^{40}$ They are also comparable with measurements on other B polyhedra like octahedra in $\mathrm{YB}_{6}$ of $1200 \pm 30$ $\mathrm{kHz}^{28}$ in $\mathrm{LaB}_{6}$ of $1028 \mathrm{kHz}^{29}$ or $\mathrm{B}_{12}$ cuboctahedra in $\mathrm{TB}_{12}(\mathrm{~T}=\mathrm{Y}$, $\mathrm{Zr}, \mathrm{Lu})$ with about $100 \mathrm{kHz} .{ }^{25}$ With this background, a comparison to the measured coupling constants of $1300 \pm 100 \mathrm{kHz}^{33}$ for $\mathrm{B}_{4} \mathrm{C}$ and $240 \mathrm{kHz}^{35}$ for rhombohedral $\alpha-\mathrm{SiB}_{3-x}$ leads to the conclusion that these may be assigned to the $\mathrm{B}$ atoms involved in the intericosahedral linkage.

For the sites $\mathrm{B}(3)$ and $\mathrm{B}(4)$ connected to the interstitial unit, the $\left|C_{\mathrm{q}}\right|$ in Table 4 clearly differ between each compound. The value of $\mathrm{B}(3)$ in $\mathrm{MgB}_{7}$ is remarkable, which indicates a coupling about $500 \mathrm{kHz}$ larger than those of $\mathrm{B}(3)$ and $\mathrm{B}(4)$ in $\mathrm{o}-\mathrm{MgB}_{12} \mathrm{C}_{2}$ with $825 \mathrm{kHz}$. For $\mathrm{B}_{4} \mathrm{C}$ literature reports $450 \pm 250 \mathrm{kHz},{ }^{33} 361$, $380,513 \mathrm{Hzk}^{34}$ as well as $840 \mathrm{kHz},{ }^{35}$ being in the range of those sites bonded to $\mathrm{C}$ in $\mathrm{o}-\mathrm{MgB}_{12} \mathrm{C}_{2}$. Also the small value of $\mathrm{B}(3)$ and the high value of $\mathrm{B}(4)$ in $\gamma-\mathrm{B}_{28}$ are noteworthy, which as we will discuss later is closely related to the electronic situation of the exohedral bond. Similar to the isotropic chemical shifts, the $\left|C_{\mathrm{q}}\right|$ of the interstitial $\mathrm{B}(5)$ atoms in $\mathrm{MgB}_{7}$ and $\gamma-\mathrm{B}_{28}$ differ significantly. Altogether we find small quadrupolar coupling constants up to $1750 \mathrm{kHz}$ for borides with icosahedral structure elements, whereas the high value of $\mathrm{B}(5)$ in $\gamma-\mathrm{B}_{28}$ is outstanding and comparable to the one of hexagonal $\mathrm{BN}$ with $3200 \mathrm{kHz} .^{35}$

The small quadrupolar asymmetry parameters $\eta_{\mathrm{q}}$ for all $\mathrm{B}(1)$ sites and for $\mathrm{B}(4)$ in $\gamma-\mathrm{B}_{28}$ reflect a high degree of symmetry for the EFG around the main axis $V_{z z}$. For the remaining positions in $\mathrm{MgB}_{7}$ and $\mathrm{o}-\mathrm{MgB}_{12} \mathrm{C}_{2}$ as well as $\mathrm{B}(3)$ we find values in the range of 0.39 and 0.54 similar to those of the equatorial sites in rhombohedral $\mathrm{B}_{12} \mathrm{P}_{2}$ and $\mathrm{B}_{12} \mathrm{As}_{2}$ with 0.47 and 0.30 , respectively. ${ }^{40}$ It is interesting that $\mathrm{B}(2)$ and $\mathrm{B}(5)$ involved in the $2 \mathrm{e} 3 \mathrm{c}$ bond in $\gamma-\mathrm{B}_{28}$ show $\eta_{\mathrm{q}} \geq 0.64$.

In addition, Table 4 contains the expected shifts for the center of gravity $\delta_{\text {cg }}$ of the central transition $(-1 / 2 \leftrightarrow 1 / 2)$ as well as the shifts obtained by conventional central transition fit $\delta_{\text {ctf }}$ of the recorded spectra (see ESI $\dagger$ for details). As specified in the footnote of Table $4, \delta_{\mathrm{q}}$ reflects the influence of the quadrupolar coupling and is also dependent on the Larmor frequency $\nu_{1}$ used in the NMR experiment. ${ }^{19,21,22}$ The effect that due to quadrupolar coupling the central transition is shifted more high-/upfield than indicated by $\delta_{\text {iso }}$ can be significant, especially in case of the $\mathrm{C}-\mathrm{B}-\mathrm{C}$ chain center atom with $C_{\mathrm{q}} \approx$ $5600 \mathrm{kHz}^{26,33}$ in $\mathrm{B}_{4} \mathrm{C} .{ }^{40}$ For the compounds investigated here, however, the smaller values of $\left|C_{\mathrm{q}}\right|$ and the applied carrier frequency of $500 \mathrm{MHz}$ lead to differences between $\delta_{\text {iso }}$ and $\delta_{\text {cg }}$ below $1.1 \mathrm{ppm}$. The only exception is the $\mathrm{B}(5)$ site in $\gamma-\mathrm{B}_{28}$, where we find $\delta_{\mathrm{q}}=3.7 \mathrm{ppm}$ due to its larger quadrupolar coupling constant, resulting in $\delta_{\mathrm{cg}}(\mathrm{B}(5))<\delta_{\mathrm{cg}}(\mathrm{B}(4))$ in contrast to $\delta_{\text {iso }}(\mathrm{B}(5))>\delta_{\text {iso }}(\mathrm{B}(4))$. Turning to $\mathrm{MgB}_{7}$ and $\mathrm{o}-\mathrm{MgB}_{12} \mathrm{C}_{2}$ we find remarkable agreement between the theoretical expected $\delta_{\text {cg }}$ and $\delta_{\text {ctf }}$ extracted from the MAS NMR measurements. The values of $\delta_{\text {ctf }}=11.1 \pm 0.8 \mathrm{ppm}$ in $\mathrm{MgB}_{7}$ and $\delta_{\text {ctf }}=-3.3 \pm$ $0.8 \mathrm{ppm}$ in $\mathrm{O}-\mathrm{MgB}_{12} \mathrm{C}_{2}$ indicate that the individual signal contributions of $\mathrm{B}(2)$ and $\mathrm{B}(3)$ as well as those of $\mathrm{B}(1)$ and $B(2)$ cannot be resolved by our experiments, respectively.

Fig. 5 and 6 show the measured MAS NMR spectra together with the contributions derived from the parameters of the allelectron PAW construction in Table 4. For the plotted spectra simulation $\delta_{\text {cg }}$ is used to set the shift position. Apart from the 
(a)

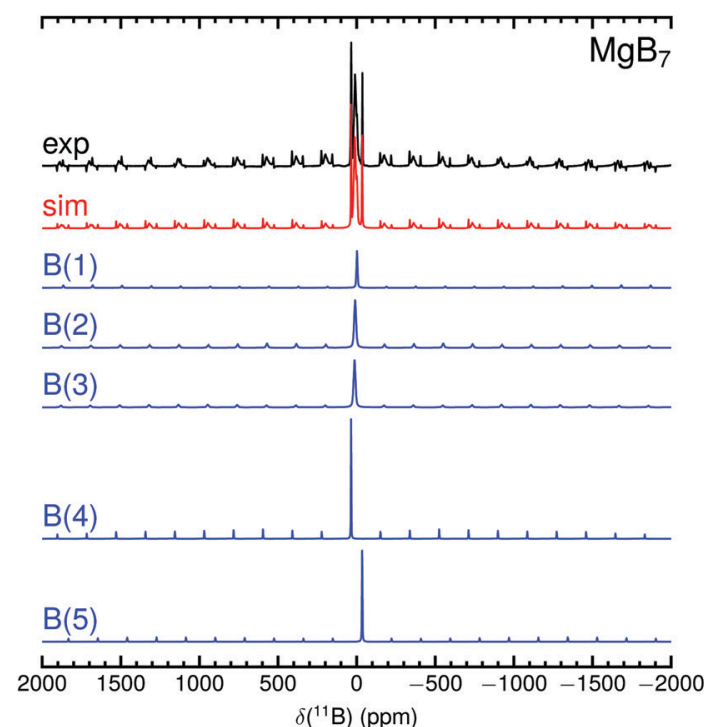

(b)

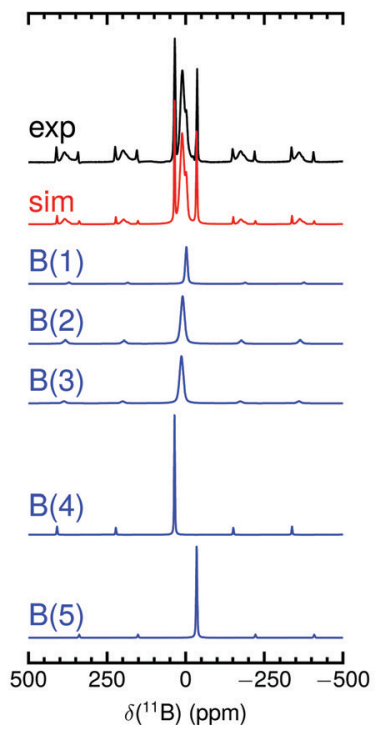

(c)

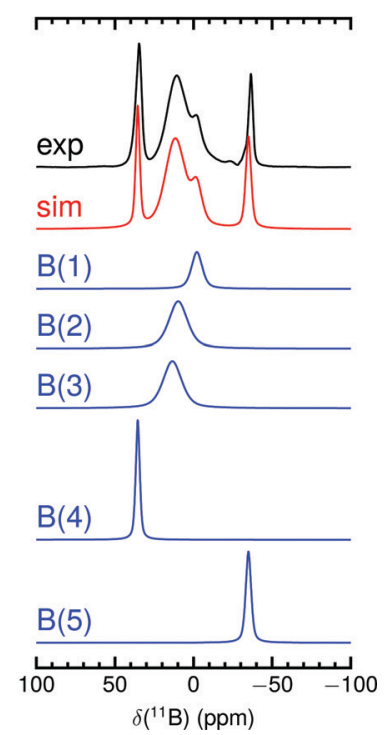

Fig. 5 Experimental ${ }^{11} \mathrm{~B}$ MAS NMR spectrum of $\mathrm{MgB}_{7}$ measured at $11.7 \mathrm{~T}$ with $30 \mathrm{kHz}$ spinning frequency and simulation with parameters from PAW calculation in Table 4 together with site contributions. $\delta_{\mathrm{cg}}$ is used in the simulation to set the shift position. Intensities and linewidths adjusted by eye for best fit of central transition shown in (c) (see Table S4 in the ESI $\dagger$ for further details).

intensity, the linewidth was adjusted by hand, taking into account the spectral shape of the central transitions only. The resulting area ratios reflect the multiplicities of $1: 2: 2: 1: 1$ in $\mathrm{MgB}_{7}$ and $1: 2: 2: 1$ in $\mathrm{o}-\mathrm{MgB}_{12} \mathrm{C}_{2}$ supposed from the symmetry positions (see Table $\mathrm{S} 4$ in ESI†). Note that in SSNMR the ratio of intensity/area of contributions does not always correspond to such expectations from site symmetry. ${ }^{30,40}$ Indeed, for boron compounds with icosahedra as structure elements it is known that extraction of reliable parameter sets from measured ${ }^{11} \mathrm{~B}$ spectra alone is difficult. ${ }^{34,35}$ With a comparison of Fig. 5 with Fig. 6 it is evident that the peak at about $-35 \mathrm{ppm}$ is caused by the interstitial $\mathrm{B}(5)$ site. However, a further assignment of signals is not readily possible and attempts of conventional fit procedure do not provide unambiguous sets of parameters for $\mathrm{MgB}_{7}$ and $\mathrm{o}-\mathrm{MgB}_{12} \mathrm{C}_{2}$.

As mentioned in the introduction the quadrupolar coupling affects the nuclear energy levels in magnetic field and therefore also the satellite transitions $( \pm 3 / 2 \leftrightarrow \pm 1 / 2)$ in measured MAS NMR. ${ }^{20}$ The simulated spectra from the calculated parameters display not only signal shape from -100 to $100 \mathrm{ppm}$ but also the sideband pattern, meaning that the computed $\left|C_{\mathrm{q}}\right|$ are also within a reasonable range of value.

$\mathrm{MgB}_{7}$ shows a shift extension from $-40 \mathrm{ppm}$ known for boronhydrides up to $30 \mathrm{ppm}$ common for borates. We are not aware of any other compound containing B with dominant covalency and significant bandgap that spans such a range. The chemical shifts of the $\mathrm{B}(2)$ and $\mathrm{B}(3)$ sites are close to one another (see Table 4) and hence their contributions in the experimental spectrum in Fig. 5 are not resolved separately. The measurement of $\mathrm{o}-\mathrm{MgB}_{12} \mathrm{C}_{2}$, on the other hand, shows a strong difference in shifts between $\mathrm{B}(2)$ and $\mathrm{B}(3)$ making two different maxima occur. In both experimental spectra the contribution of $\mathrm{B}(4)$ is clearly visible near $35 \mathrm{ppm}$.
As the current review by AngelWong and Bryce ${ }^{19}$ reports, complete NMR parameter sets are known for only very few boron-rich borides based on boron polyhedra. Of particular interest here might be compounds $\mathrm{T}^{\prime} \mathrm{B}_{n}\left(\mathrm{~T}^{\prime}=\mathrm{Y}, \mathrm{La} ; n=4,6\right)$ which are composed of $\mathrm{B}_{6}$ octahedra incorporating $\mathrm{B}_{2}$ dumbbells for $n=4\left(\mathrm{~T}_{2}^{\prime}{ }_{2}\left[\mathrm{~B}_{6}\right]\left[\mathrm{B}_{2}\right]\right)$. At least structurally there is a similarity to the transition from $\mathrm{B}_{12}$ icosahedra as in $\alpha-\mathrm{rB}_{12}$ to polyhedra additionally linked by an interstitial unit as in $\mathrm{MgB}_{7}$ and $\gamma-\mathrm{B}_{28}$. Experimental reports assign for $\mathrm{YB}_{4}{ }^{11} \mathrm{~B}$ shifts in the range of 5.4 to $34.7 \mathrm{ppm}^{62}$ and for $\mathrm{LaB}_{4}$ of 18 to $47 \mathrm{ppm}^{24}$

In the transition from $\mathrm{TB}_{6}$ to $\mathrm{TB}_{4}$, the $C_{\mathrm{q}}$ of the atoms connected to the dumbbell are close to those interconnecting the octahedra. For the $4 \mathrm{e}$ and $8 \mathrm{j}$ sites in $\mathrm{YB}_{4}$ (correspond to $\mathrm{B}(1)$ and $\mathrm{B}(3) / \mathrm{B}(4))$ a value of Grindeq issue $1030 \pm 60 / 80 \mathrm{kHz}^{28}$ is observed each and in $\mathrm{LaB}_{4} 690$ and $800 \mathrm{kHz}$ are reported for $4 \mathrm{e}$ and $8 \mathrm{j} .{ }^{24}$ The values of $\mathrm{YB}_{6}$ and $\mathrm{LaB}_{6}$ are $1200 \pm 30$ and $1100 \mathrm{kHz}{ }^{28,29}$ respectively. This observation is also true for $\mathrm{B}(3)$ in $\mathrm{MgB}_{7}$ and $\mathrm{B}(4)$ in $\gamma-B_{28}$, but should not be generalized, because on one hand it is not valid for $\mathrm{B}(4)$ in $\mathrm{MgB}_{7}$ and $\mathrm{B}(2)$ in $\gamma-\mathrm{B}_{28}$ and on the other hand an unchecked general prediction of the quadrupolar interaction and, thus, also of the EFG among different boron polyhedra and arrangements should be done with care. The case of $\mathrm{CaB}_{4-x} \mathrm{C}_{x}$ $(0.21 \leq x \leq 0.26)$ illustrates how problematic the extraction of NMR parameters by fitting procedures of measured spectra solely is. There only the $8 \mathrm{j}$ position with $11 \mathrm{ppm}$ and $800 \mathrm{kHz}$ could be assigned. The two remaining sites (4e and $4 \mathrm{~h}$ ) showed satellite transitions with too large linewidth and, therefore, too low intensity in order to determine any isotropic chemical shift or quadrupolar coupling constant. ${ }^{24}$

The shifts of the $\mathrm{B}_{2}$ dumbbell in Table 4 remarkably differ not only for $\mathrm{MgB}_{7}$ and $\gamma-\mathrm{B}_{28}$, but also in $\mathrm{YB}_{4}$ with $12.6 \mathrm{ppm}^{62}$ and $42 \mathrm{ppm}$ in $\mathrm{LaB}_{4} \cdot{ }^{24}$ Overall, caution is required when comparing 
(a)



(b)

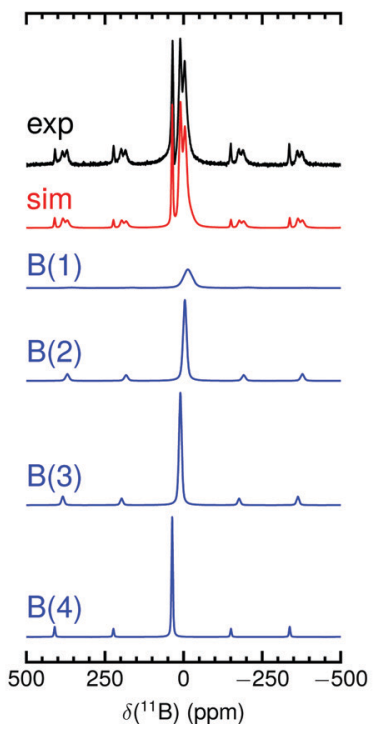

(c)

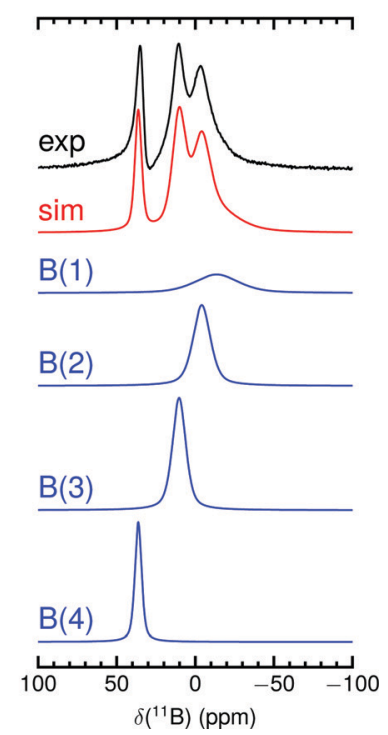

Fig. 6 Experimental ${ }^{11} \mathrm{~B}$ MAS NMR spectrum of $\mathrm{o}-\mathrm{MgB}_{12} \mathrm{C}_{2}$ measured at $11.7 \mathrm{~T}$ with $30 \mathrm{kHz}$ spinning frequency and simulation with parameters from PAW calculation in Table 4 together with site contributions. $\delta_{\mathrm{cg}}$ is used in the simulation to set the shift position. Intensities and linewidths adjusted by eye for best fit of central transition shown in (c) (see Table S4 in the ESI† for further details).

these structures, because the significant electron density around the Fermi level due to the transition metals has an influence on the magnetic shielding of $\mathrm{B}$. This is especially true for the dumbbell atoms, which, similar to Fig. $4 \mathrm{a}$ and c, contribute significantly to the DOS at this area but, in addition, their p-states extend continously above $0 \mathrm{eV}$ and, thus, interacting with the metallic bands. ${ }^{29,62,70}$ Besides the influence of the electrons from the valence band (orbital contribution), there is an additional contribution from the interaction of spin-polarized electrons from the conduction band with the magnetic moments of the B nuclei, which is also well known as Knight shift for metallic systems. Thus, we want to emphasize that the significant bandgaps in Fig. 4 are of major importance for the chemical shifts in Table 4 to be reasonable because they are calculated with relying on the orbital contribution of the chemical shielding tensor, ${ }^{37,38}$ only. Eventually, considering this contribution is sufficient when systems under investigation are nonmagnetic semiconductors without unpaired electrons or partially filled bands at the Fermi surface. Plane wave approaches to calculate the Knight shift had been developed within the GIPAW $^{65}$ as well as the LAPW ${ }^{71}$ method, and had been applied for example to several Heusler phases. ${ }^{72,73}$

\subsection{Analysis of the EFG}

Since the EFG is a tensor evaluated at the location of the nucleus, the orientation of its main axes is limited to the corresponding spatial symmetry. As explained above, in the compounds investigated here a mirror plane $\sigma_{\mathrm{h}}$ runs through the $\mathrm{B}(1), \mathrm{B}(4)$ and interstitial atoms. Consequently, there is a $C_{\mathrm{s}}$ point symmetry at those positions. This means for $\mathrm{B}(1), \mathrm{B}(4)$ and $\mathrm{B}(5)$ two axes of the EFG must lie in $\sigma_{\mathrm{h}}$ with the third being perpendicular to the plane. Note that this consideration does not apply strictly to the positions of $\mathrm{B}(2)$ and $\mathrm{B}(3)$ with $C_{1}$ symmetry.

Table 5 informs about the orientation of the large EFG axis $V_{z z}$ by the inclined angles relative to the $\mathrm{B}_{12}$ icosahedron defined in Fig. 7. For comparison, $\left(\mathrm{B}_{12} \mathrm{H}_{12}\right)^{2-}$ is added with its closely related structure of ideal $I_{\mathrm{h}}$ symmetry. ${ }^{74}$ The mentioned symmetry condition for $\mathrm{B}(1), \mathrm{B}(4)$ and $\mathrm{B}(5)$ leads to the result that the sum of two of the three angles $\varphi, \Theta_{V_{z z}}$ and $\Phi_{V_{z z}}$ must add up to the remaining angle. As in the rhombohedral boron-rich phases ${ }^{40}$ we also observe here the general tendency of $V_{z z}$ for $\mathrm{B}(1)$ to $\mathrm{B}(4)$ to align either along the bond or radially, that is minimizing $\Theta_{V_{z z}}$ or $\Phi_{V_{z z}}$. This can be expected due to the dominance of the $2 \mathrm{e} 2 \mathrm{c}$ exohedral bond throughout the investigated compounds as previously described and to the finding that B polyhedra contain almost no charge. ${ }^{7}$ Note that there are also cases of icosahedral $B_{12}$ arrangements were this does not hold true, as for example in carboranes ${ }^{32}$ or in $\mathrm{B}_{12} \mathrm{As}_{2}{ }^{40}$

A remarkable feature, that will become central for the relation between the magnitude of $V_{z z}$ and the exohedral bonding situation, is the special geometrical arrangement of the here investigated polyhedra. The apex angles $\theta$ and $\theta^{\prime}$ of the regular icosahedron are such that the influence of endohedral bonding partners on $V_{z z}$ can be assumed of minor contribution and to a good approximation its value mainly reflects the exohedral bonding situation. The key condition of this approximation is that $\left(\theta_{\mathrm{av}}+\theta_{\mathrm{av}}^{\prime}\right) / 2 \approx \theta_{\mathrm{mag}}$ is valid with $\theta_{\mathrm{mag}}$ as magic angle fulfilling $\cos \theta_{\text {mag }}=1 / \sqrt{3}$. Therefore, Table 5 additionally shows the absolute deviation from the magic angle as $\Delta \bar{\theta}=\left|\left(\theta_{\mathrm{av}}+\theta_{\mathrm{av}}^{\prime}\right) / 2-\theta_{\mathrm{mag}}\right|$. This spatial property is derived and discussed in detail in ref. 40. There, a particular B site in rhombohedral boron-rich phases for which the exohedral 
Table 5 Angles in ${ }^{\circ}$ related to the orientation of the large $V_{z z}$ axis of the EFG and to structure geometry with average values $\theta_{\mathrm{av}}$ and $\theta_{\mathrm{av}}^{\prime}$ (see Fig. 7 for definition). $\Delta \bar{\theta}$ denotes the absolute mean deviation from the magic angle $\theta_{\text {mag }}\left(\approx 54.74^{\circ}\right)$ defined as $\left|\left(\theta_{\mathrm{av}}+\theta_{\mathrm{av}}^{\prime}\right) / 2-\theta_{\mathrm{mag}}\right|$. All values according to PAW PPs and optimized structures

\begin{tabular}{|c|c|c|c|c|c|c|c|}
\hline \multirow[b]{2}{*}{ Compound } & \multirow[b]{2}{*}{ Site } & \multicolumn{2}{|c|}{ EFG related } & \multicolumn{4}{|c|}{ Geometry related } \\
\hline & & $\Theta_{V_{z z}}$ & $\Phi_{V_{z z}}$ & $\varphi$ & $\theta_{\mathrm{av}}$ & $\theta_{\mathrm{av}}^{\prime}$ & $\Delta \bar{\theta}$ \\
\hline \multirow[t]{5}{*}{$\mathrm{MgB}_{7}$} & $\mathrm{~B}(1)$ & 0.29 & 6.79 & 6.50 & 57.52 & 51.79 & 0.08 \\
\hline & $\mathrm{B}(2)$ & 10.65 & 23.47 & 22.63 & 57.69 & 51.99 & 0.10 \\
\hline & $\mathrm{B}(3)$ & 6.51 & 12.41 & 8.98 & 58.51 & 52.87 & 0.95 \\
\hline & $\mathrm{B}(4)$ & 12.30 & 0.99 & 11.31 & 59.77 & 54.23 & 2.26 \\
\hline & $\mathrm{B}(5)$ & & $6.57^{a}$ & & & & \\
\hline \multirow[t]{4}{*}{$\mathrm{o}-\mathrm{MgB}_{12} \mathrm{C}_{2}$} & $\mathrm{~B}(1)$ & 5.47 & 0.25 & 5.71 & 60.05 & 54.54 & 2.56 \\
\hline & $\mathrm{B}(2)$ & 7.55 & 21.30 & 21.84 & 58.62 & 53.00 & 1.07 \\
\hline & $\mathrm{B}(3)$ & 6.32 & 4.74 & 7.84 & 57.19 & 51.45 & 0.42 \\
\hline & $\mathrm{B}(4)$ & 8.08 & 16.52 & 8.44 & 58.01 & 52.34 & 0.44 \\
\hline \multirow{7}{*}{$\gamma-\mathrm{B}_{28}$} & $\mathrm{~B}(1)$ & 2.18 & 6.64 & 4.46 & 59.11 & 53.51 & 1.57 \\
\hline & $\mathrm{B}(2)^{b}$ & 12.31 & 17.76 & 11.14 & 58.13 & 52.45 & 0.55 \\
\hline & & $72.09^{c}$ & & $54.62^{c}$ & & & \\
\hline & $\mathrm{B}(3)^{b}$ & 37.56 & 21.72 & 20.02 & 57.61 & 51.89 & 1.56 \\
\hline & & $9.60^{c}$ & & $16.33^{c}$ & & & \\
\hline & $\mathrm{B}(4)$ & 9.95 & 2.63 & 7.32 & 59.10 & 53.50 & 0.71 \\
\hline & $\mathrm{B}(5)$ & & $33.54^{a d}$ & & & & \\
\hline \multicolumn{2}{|l|}{$\left(\mathrm{B}_{12} \mathrm{H}_{12}\right)^{2-}$} & 0.02 & 0.02 & 0.00 & 58.28 & 52.62 & 0.71 \\
\hline
\end{tabular}

${ }^{a}$ Relative to $\mathrm{B}(5)-\mathrm{B}(5)$ direction. ${ }^{b}$ Along exohedral $\mathrm{B}(2)-\mathrm{B}(3)$ direction. ${ }^{c}$ Relative to exohedral $\mathrm{BCP}$ of $\mathrm{B}(2)-\mathrm{B}(5)$ bond (see Fig. 8). ${ }^{d}$ Relative to $\mathrm{B}(4)-\mathrm{B}(5)$ direction $30.13^{\circ}$. (a)

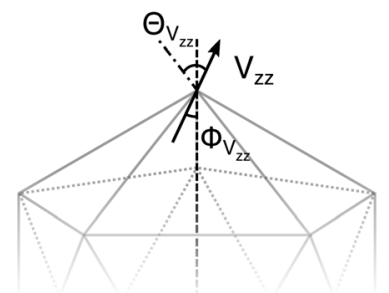

(b)

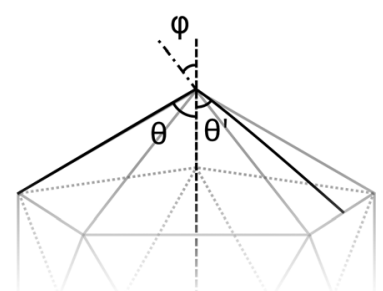

Fig. 7 Definition of angles related to (a) the main axis of the EFG $V_{z z}$ and (b) structure geometry. The dash dotted line points to exohedral bonding partner, dashed line represents radial direction from icosahedral center of mass.

bond being the strongest, $V_{z z}$ is radially oriented $\left(\Phi_{V_{z z}}<\Theta_{V_{z z}}\right)$, if $\Delta \bar{\theta}>2^{\circ}$. Here, we find that the $V_{z z}$ axis aligns in a good approximation with the exohedral bond as $\Delta \bar{\theta} \leq 2^{\circ}$ but for higher deviations as in the case of the $\mathrm{B}(4)$ site in $\mathrm{MgB}_{7}$ and the $\mathrm{B}(1)$ site in $\mathrm{o}-\mathrm{MgB}_{12} \mathrm{C}_{2}$ the $V_{z z}$ axis is pointing radially. Note that it is not appropriate to reverse this observation, in particular that small $\Delta \bar{\theta}$ do not necessarily lead to an alignment with the exohedral bond as can be seen by the examples of $\mathrm{B}(3)$ in $\mathrm{o}-\mathrm{MgB}_{12} \mathrm{C}_{2}$ or $\mathrm{B}(4)$ in $\gamma-\mathrm{B}_{28}$. Certainly there are also other influences on the $V_{z z}$ orientation such as the positive charge of $\mathrm{Mg}$ in $\mathrm{MgB}_{7}$ and $\mathrm{o}-\mathrm{MgB}_{12} \mathrm{C}_{2}$. However, assuming these effects is very involved and we do not consider them mandatory for the herein given discussion.

Fig. 8 shows the orientations of the $V_{z z}$ axes for $\mathrm{B}(2)$ and $\mathrm{B}(3)$ in the vicinity of the $\mathrm{B}(5)$ site and its (exohedral) bonds of $\gamma-\mathrm{B}_{28}$.

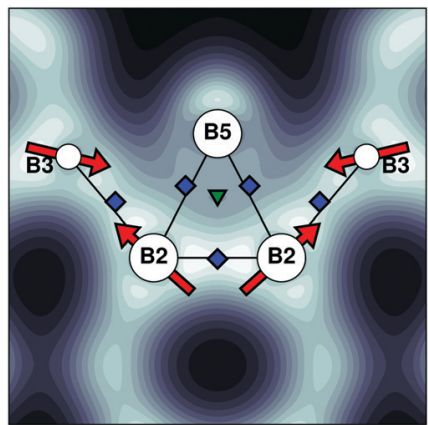

Fig. $8 \quad V_{z z}$ vectors (arrows) of $\mathrm{B}(2)$ and $\mathrm{B}(3)$ sites projected on the $\mathrm{B}(2)-$ $B(2)-B(5)$ plane of $\gamma-B_{28}$ together with BCPs (diamond) and RCP (triangle) from Bader's QTAIM analysis. Underlying contour map corresponds to the calculated electron charge density depicted in Fig. 3 a.

We want to note here again that there is no strict condition for the $V_{z z}$ alignment of these sites according to symmetry considerations. Although for both $\mathrm{B}(2)$ and $\mathrm{B}(3)$ the $\mathrm{BCP}$ of $\mathrm{B}(2)-\mathrm{B}(3)$ is the closest, $V_{z z}$ of $\mathrm{B}(3)$ is oriented to the $\mathrm{BCP}$ of the $\mathrm{B}(2)-\mathrm{B}(5)$ bonding path. As it is evident from an inspection of Table 3 the magnitude of the electron density at this BCP cannot be the reason for the shown orientation on $\mathrm{B}(3)$. In this regard, one has to keep in mind that the relative position of the $B_{2}$ unit in $\gamma-B_{28}$ changes the exohedral electronic situation for the $\mathrm{B}(2)$ and $\mathrm{B}(3)$ sites. This is in contrast to all icosahedral sites in $\mathrm{MgB}_{7}$ and $\mathrm{o}-\mathrm{MgB}_{12} \mathrm{C}_{2}$ with distinct $5+1$ coordination.

A comparison of the possible kink angles $\varphi$ in Table 5 to the respective BCPs shows how the $V_{z z}$ axes point to those BCPs which have a smaller deviation from the polyhedral center with vanishing electron density. In particular, $\varphi$ for $\mathrm{B}(2)$ with $11.14^{\circ}$ is much smaller towards $\mathrm{B}(3)$ than with $54.62^{\circ}$ pointing towards $\mathrm{B}(5)$. In fact, if $V_{z z}$ is oriented to the $\mathrm{BCP}$ of $\mathrm{B}(2)-\mathrm{B}(5)$, the influence of the electron density of the endohedral bonds would increase and consequently the gradient of the charge distribution would decrease (see Fig. 8). This means in turn, however, that such an orientation does no longer represent the largest EFG at $\mathrm{B}(2)$. A similar situation can be found for $\mathrm{B}(3)$, where $V_{z z}$ points to the $\mathrm{BCP}$ of the $\mathrm{B}(2)-\mathrm{B}(5)$ bond. With an alignment to the exohedral $\mathrm{B}(2)-\mathrm{B}(3)$ bond a decrease of the gradient can be expected due to the electron density of the endohedral bonds as illustrated in Fig. 8. In $\gamma-\mathrm{B}_{28}$ the exohedral bonding situation for $\mathrm{B}(2)$ is modified by the interaction to the interstitial $\mathrm{B}_{2}$ dumbbell compared to $\mathrm{MgB}_{7}$ and $\mathrm{o}-\mathrm{MgB}_{12} \mathrm{C}_{2}$ in such way that $V_{z z}$ of $\mathrm{B}(3)$ is not aligned to its exohedral bonding partner $\mathrm{B}(2)$.

For the $\mathrm{B}(5)$ positions of the interstitial $\mathrm{B}_{2}$ units in $\mathrm{MgB}_{7}$ and $\gamma-\mathrm{B}_{28}$ we observe different orientations of $V_{z z}$. While in $\mathrm{MgB}_{7}$ the $V_{z z}$ axis points along the bond within the dumbbell, in $\gamma-\mathrm{B}_{28}$ it is tilted in such a way that it can be associated neither with the direction of $\mathrm{B}(4)-\mathrm{B}(5)$ nor along $\mathrm{B}(5)-\mathrm{B}(5)$.

An overview of the full set of EFG components for all B sites can be found in Table 6. The values of the PPs from the NC and PAW approach differ only slightly. Altogether, the icosahedral atoms show negative $V_{z z}$ values, which in combination with the alignments of the corresponding axes in Table 5 demonstrates 
Table 6 EFG tensor components in $10^{20} \mathrm{~V} \mathrm{~m}^{-2}$ determined by $\mathrm{NC}$ and PAW type PPs

\begin{tabular}{|c|c|c|c|c|c|c|c|}
\hline \multirow[b]{2}{*}{ Compound } & \multirow[b]{2}{*}{ Site } & \multicolumn{3}{|l|}{$\mathrm{NC}$} & \multicolumn{3}{|l|}{ PAW } \\
\hline & & $V_{x x}$ & $V_{y y}$ & $V_{z z}$ & $V_{x x}$ & $V_{y y}$ & $V_{z z}$ \\
\hline \multirow{5}{*}{$\mathrm{MgB}_{7}$} & $\mathrm{~B}(1)$ & 5.9 & 8.2 & -14.2 & 5.7 & 7.9 & -13.7 \\
\hline & $\mathrm{B}(2)$ & 2.0 & 6.8 & -8.8 & 2.0 & 6.6 & -8.6 \\
\hline & $\mathrm{B}(3)$ & 3.6 & 9.5 & -13.1 & 3.5 & 9.3 & -12.8 \\
\hline & $\mathrm{B}(4)$ & 2.5 & 6.7 & -9.2 & 2.4 & 6.5 & -8.9 \\
\hline & $\mathrm{B}(5)$ & -4.3 & -11.0 & 15.3 & -4.2 & -10.8 & 14. \\
\hline \multirow[t]{4}{*}{$\mathrm{o}-\mathrm{MgB}_{12} \mathrm{C}_{2}$} & $\mathrm{~B}(1)$ & 8.0 & 9.3 & -17.3 & 7.7 & 9.0 & -16.7 \\
\hline & $\mathrm{B}(2)$ & 2.3 & 5.4 & -7.7 & 2.3 & 5.3 & -7 \\
\hline & $\mathrm{B}(3)$ & 2.5 & 6.2 & -8.7 & 2.5 & 5.9 & -8.4 \\
\hline & $\mathrm{B}(4)$ & 2.1 & 6.3 & -8.4 & 2.1 & 6.1 & -8.2 \\
\hline \multirow[t]{5}{*}{$\gamma-B_{28}$} & $\mathrm{~B}(1)$ & 9.0 & 9.2 & -18.2 & 8.7 & 9.0 & -17.6 \\
\hline & $\mathrm{B}(2)$ & 0.6 & 4.0 & -4.7 & 0.7 & 3.8 & -4 \\
\hline & $\mathrm{B}(3)$ & 1.0 & 2.7 & -3.7 & 1.0 & 2.7 & -3.7 \\
\hline & $\mathrm{B}(4)$ & 8.8 & 9.7 & -18.5 & 8.5 & 9.5 & -17.9 \\
\hline & $\mathrm{B}(5)$ & 6.3 & 28.3 & -34.6 & 5.9 & 27.2 & -33.1 \\
\hline$\left(\mathrm{B}_{12} \mathrm{H}_{12}\right)^{2-}$ & & $4.3^{a}$ & & -8.5 & $4.1^{a}$ & & -8 . \\
\hline
\end{tabular}

that especially the electronic situation of the exohedral bonds is significant. Accordingly, a correlation between the bond strength, that is in terms of Bader's analysis the density value at the exohedral BCP (see Table 3), and $V_{z z}$ could be suspected. And as the plot (Fig. S1 of ESI $\dagger$ ) indicates, the higher the charge $\rho\left(r_{\mathrm{BCP}}\right)$ is, the more negative the values of $V_{z z}$ become for the icosahedral sites. Strictly speaking, this does not apply over all compounds but we obtain an approximately linear relationship within each system.

To further elucidate the values given in Table 6 we point out that $V_{z z}=\int \rho(r) \frac{2 P_{2}(\cos \vartheta)}{r^{3}} \mathrm{~d}^{3} r$ holds within the principal axis system of the EFG tensor where $\mathrm{P}_{2}$ is the second-order Legendre polynomial. As Schwarz et $a .^{26}$ explain, after decomposing this expression in the lattice $V_{z z}^{\text {lat }}$ and spherical part $V_{z z}^{l^{\prime \prime}}$ the contribution of particular angular momenta can be analyzed. In doing so, the portion of the p orbitals with $V_{z z}^{\mathrm{pp}} \propto \Delta n_{\mathrm{p}_{z}}\left\langle\frac{1}{r^{3}}\right\rangle_{\mathrm{p}}$ turns out to be the main component for borides containing $B_{6}$ octahedra. ${ }^{25,28,29}$ For B atoms in $\mathrm{B}_{12}$ icosahedra in $\alpha-\mathrm{rB}_{12}$ and $\mathrm{B}_{4} \mathrm{C}$ this contribution to $V_{z z}$ in total accounts for even more than $80 \% .{ }^{26}$ Apart from the factor $\left\langle 1 / r^{3}\right\rangle_{\mathrm{p}}$ which is about $0.69 \AA$ for these compounds, also the magnitude of the so-called anisotropy count $\Delta n_{\mathrm{p}_{z}}=\left(n_{\mathrm{p}_{x}}+n_{\mathrm{p}_{y}}\right) / 2-n_{\mathrm{p}_{z}}$ with partial charges $n_{\mathrm{p}_{i}}$ in the corresponding $\mathrm{p}$ channels is mandatory.

If we assume that the electron excess between the averaged occupation of $\mathrm{p}_{x}$ and $\mathrm{p}_{y}$ is nearly constant compared to $\mathrm{p}_{z}$ for the icosahedral B atoms considered here, it follows that $V_{z z}^{\mathrm{pp}} \propto$ $\left\langle 1 / r^{3}\right\rangle_{\mathrm{p}}$. Motivated by this and the fact that the $V_{z z}$ axes are aligned along the exohedral bonds, we plot in Fig. 9 the properties from QTAIM analysis for exohedral bonds in Table 3 with $\rho\left(r_{\mathrm{BCP}}\right) / d_{\mathrm{X}-\mathrm{BCP}}{ }^{3}$ against the corresponding $V_{z z}$ components. In contrast to Fig. S1 of the ESI, $\dagger$ not only the strength of the bond is characterized by $\rho\left(r_{\mathrm{BCP}}\right)$ but also the



Fig. 9 Values of the EFG main component $V_{z z}$ from PAW calculations plotted against $\rho\left(r_{\mathrm{BCP}}\right) / d_{\mathrm{X}-\mathrm{BCP}}{ }^{3}$ of $\mathrm{B}$ sites $\mathrm{B}(1)$ to $\mathrm{B}(4)$ in the icosahedral $\mathrm{B}_{12}$ units with data from Tables 3 and 6 and of polar atoms $B(1)$ from the first part ${ }^{40}$ of this publication series (denoted as others). The properties of the BCPs are associated with the exohedral bonds and in accordance to the orientation of the $V_{z z}$ axis. ${ }^{75}$ Filled symbols correspond to properties of sites bonded to the interstitial unit. The trend line is meant to guide the eye.

influence of the distance of the accorded BCP to the atom is taken into account. As shown, there is a clear linear relationship and therewith also for measurable $C_{\mathrm{q}} / \nu_{\mathrm{q}}$ values of icosahedral B atoms with an exohedral homonuclear bond. This trend is consistent throughout the compounds investigated here and is also valid for polar positions in $\alpha-\mathrm{rB}_{12}$ and $\mathrm{B}_{12} \mathrm{X}_{2}$ $(\mathrm{X}=\mathrm{P}, \mathrm{As}, \mathrm{O})$ with intericosahedral linkage ${ }^{40}$ denoted as others in Fig. 9.

We want to emphasize that this correlation inherits a very strong simplification, which is not at least valid due to the special polyhedral geometry with satisfying $\left(\theta_{\mathrm{av}}+\theta_{\mathrm{av}}^{\prime}\right) / 2 \approx \theta_{\mathrm{mag}}$. This results in the crucial importance of the electronic situation along the exohedral bond for values of $V_{z z}$. In fact, a comparison of the $d_{\mathrm{X}-\mathrm{BCP}}$ in Table 3 and for the rhombohedral phases in ref. 40 reveals that the majority of BCPs of the exohedral bonds are in range of $0.85 \AA$ and, thus, close to the previously mentioned radius of $0.69 \AA$, in which more than $80 \%$ of the EFG components originate from the contribution of the p orbitals. We believe that this observation additionally supports the illustrated relationship in Fig. 9, since the BCPs from QTAIM analysis are close to this distance. The case of $\gamma-B_{28}$ is interesting in this context. The remarkably small $V_{z z}$ value of $\mathrm{B}(2)$ seems to correspond to the $1 \mathrm{e} 2 \mathrm{c} B(2)-\mathrm{B}(3)$ bond with low density. In contrast, we suppose for $\mathrm{B}(3)$ that the distance of $1.674 \AA$ to the $\mathrm{BCP}$ on the $\mathrm{B}(2)-\mathrm{B}(5)$ bonding path is essentially responsible for the even smaller value of $-3.7 \times 10^{20} \mathrm{~V} \mathrm{~m}^{-2}$.

However, the presented approximation is no more valid for changes in $\Delta n_{\mathrm{p}_{z}}$ caused by a considerable bond polarization. This is indicated as a large shift of the exohedral BCP from QTAIM analysis for icosahedral $B$ atoms. In the case of the equatorial $\mathrm{B}$ atoms in $\mathrm{B}_{12} \mathrm{X}_{2}(\mathrm{X}=\mathrm{P}, \mathrm{As}, \mathrm{O})$, that are bonded to interstitial non-boronic atoms, we consequently find large variations in value and sign of $V_{z z}$. Furthermore, these fluctuations result in high deviations (not explicitly shown) from the plotted trend line in Fig. 9. 
Regarding $\mathrm{o}-\mathrm{MgB}_{12} \mathrm{C}_{2}, \mathrm{~B}(3)$ and $\mathrm{B}(4)$ are in a polar covalent bonding situation to $\mathrm{C}$ and accordingly $d_{\mathrm{B}-\mathrm{BCP}}$ significantly decreases compared to all other exohedral bonds. With simply considering Fig. 9 one could therefore superficially expect much more negative $V_{z z}$ than those of Table 6. However, the more electronegative $\mathrm{C}$ decreases the electronic population of the $\mathrm{p}_{z}$ orbital in the vicinity of the $\mathrm{B}$ atoms. This reduces the excess $\Delta n_{z}$ and also the absolute values of $V_{z z}$ compared to $\mathrm{MgB}_{7}$. The underlying assumption here is that in general the exohedral bonds are stronger than the endohedral ones within all icosahedra. Finally, we want to note that values of $-8.2 \times 10^{20}$ and $-8.4 \times 10^{20} \mathrm{~V} \mathrm{~cm}^{-2}$ computed with PAW agree well with $-6.9 \times 10^{20} \mathrm{~V} \mathrm{~cm}^{-2}$ calculated for B sites in a similar bonding situation at equatorial positions in $\mathrm{B}_{4} \mathrm{C} .^{26,27}$

We see a close connection of the present work with the study of Mondal et al. ${ }^{15}$ on the bonding situation in $\alpha-\mathrm{rB}_{12}$ and $\gamma-\mathrm{B}_{28}$ proposing that the orbital order of the 12 external bonding orbitals of the $B_{12}$ unit persists even if the polyhedra are involved in different exohedral bonds. With the here presented results and those of our previous investigation ${ }^{40}$ we suppose that this orbital order might be related to the orientation and magnitude of the large main axis $V_{z z}$ of the EFG and, hence, to measurable quadrupole coupling constants $C_{\mathrm{q}}$ or frequencies $\nu_{\mathrm{q}}$ of icosahedral B atoms in boron-rich borides.

For the $\mathrm{B}(5)$ site in $\mathrm{MgB}_{7}$ we find the $V_{z z}$ axis pointing along the interstitial bond with a positive value which means a change in sign of $\Delta n_{\mathrm{p}_{z}}$ in comparison to the icosahedral sites. This is in line with the values of $\rho\left(r_{\mathrm{BCP}}\right)$ in range of 0.832 to $0.905 \mathrm{eV}^{-3}$ indicating that the three $\mathrm{B}(5)-\mathrm{B}(12)$ bonds show higher electron population and are, thus, stronger than the $\mathrm{B}(5)-\mathrm{B}(5)$ interaction $\left(0.420 \mathrm{eV} \AA^{-3}\right.$, see Table 3). Contrary, in $\gamma-\mathrm{B}_{28}$ the increased valence electron density along $\mathrm{B}(4)-\mathrm{B}(5)$ is probably responsible for the negative $V_{z z}$ value. However, the orientation of the axis is less distinct. The electronic situation to the $\mathrm{B}(2)$ site but more important to the other $\mathrm{B}(5)$ dumbbell atom does have an influence. With the shorter interatomic distance of $1.729 \AA$ and $\rho\left(r_{\mathrm{BCP}}\right)=0.980 \mathrm{eV} \AA^{-3}$ we consider an increase of bond strength compared to $\mathrm{B}(5)-\mathrm{B}(5)$ in $\mathrm{MgB}_{7}$ leading to the observed tilt of the $V_{z z}$ axis.

\section{Conclusion}

In conclusion, we have discussed the structure, bonding situation and calculated tensor parameters of the chemical shift and quadrupole coupling for the orthorhombic phases $\mathrm{MgB}_{7}$, $\mathrm{o}-\mathrm{MgB}_{12} \mathrm{C}_{2}$ and $\gamma-\mathrm{B}_{28}$. Both, the similarities and differences of the exohedral icosahedral bonds as well as those of the interstitial dumbbell units with regard to the DOS were additionally examined.

The values determined by computations using the GIPAW approach were applied to simulate MAS ${ }^{11} \mathrm{~B}$ NMR spectra and compared to measurements of $\mathrm{MgB}_{7}$ and $\mathrm{o}-\mathrm{MgB}_{12} \mathrm{C}_{2}$. With this at hand, we successfully explain the measured spectra and clearly assign individual signal contributions to corresponding B sites. In combination with our previous results for rhombohedral phases ${ }^{40}$ we are confident of additional presenting appropriate NMR parameters of the orthorhombic boron phase $\gamma-B_{28}$. Overall, the comparison between results from the PP construction schemes of NC and PAW shows only small deviations.

The NMR parameters for atoms of the $\mathrm{B}_{2}$ dumbbell in $\mathrm{MgB}_{7}$ and $\gamma-\mathrm{B}_{28}$ notably differ and show hardly any similarity to those of borides containing $\mathrm{B}_{6}$ octahedra such as $\mathrm{YB}_{4}, \mathrm{LaB}_{4}$ or $\mathrm{CaB}_{4-x} \mathrm{C}_{x}$. This is due to different bonding distances within the $\mathrm{B}_{2}$ units and also due to the peculiar bonding situation to the boron polyhedra. Regarding $\mathrm{YB}_{4}, \mathrm{LaB}_{4}$ and $\mathrm{CaB}_{4-x} \mathrm{C}_{x}$ another certain reason is the influence of electrons from the (transition)metal atoms on the shielding of the B atoms. For the here investigated compounds, the $\mathrm{Mg}$ atoms play only an indirect role for the ${ }^{11} \mathrm{~B}$ shifts due to their almost complete electronic charge transfer, while it is for instance already known for Ca that its deep lying $3 \mathrm{~d}$ shells may have a significant impact on oxidic shieldings by partially covalent $\mathrm{Ca}-\mathrm{O}$ bonds in oxides and aluminosilicates. ${ }^{76}$ Furthermore, the semiconducting character indicated by the significant bandgaps ensures a very good agreement of the here reported experimental observations with the ${ }^{11} \mathrm{~B}$ shifts computed by orbital contribution to the magnetic shielding only. Yet, for metallic systems with unpaired electrons or partially filled bands at the Fermi surface the electronic spin contribution must be additionally considered as well for reliable results.

The EFG main axis $V_{z z}$ of atoms in the $\mathrm{B}_{12}$ icosahedra points to a good approximation along the stretched exohedral bond. This observation leads to a linear relation between values of $V_{z z} / C_{\mathrm{q}} / \nu_{\mathrm{q}}$ and properties of BCPs from Bader's analysis; With increasing ratio of the electron density at the BCP to its cubic distance to the respective $\mathrm{B}$ atom, the strength of the quadrupole coupling also increases. The underlying reason for this correlation is the great similarity of the exohedral $\mathrm{B}-\mathrm{B}$ bonds and the close structural relationship, that is the $\mathrm{B}_{12}$ icosahedron as main building block, of the investigated compounds. For such bonds as $\mathrm{B}-\mathrm{C}$ in $\mathrm{o}-\mathrm{MgB}_{12} \mathrm{C}_{2}$ and $\mathrm{B}-\mathrm{X}(\mathrm{X}=\mathrm{P}, \mathrm{As}, \mathrm{O})$ in rhombohedral phases, however, this statement cannot be confirmed because their bond polarity exceeds the boundaries of this quite simplified model. Concerning the quadrupolar asymmetry $\eta_{\mathrm{q}}$ there is a correspondence to the electronic bonding situation. While computed values are below 0.20 for B sites involved in $2 \mathrm{e} 2 \mathrm{c}$ bonds of the intericosahedral linkage along the hexagonal rods and commonly between 0.40 and 0.55 for those in interstitial linkage, those of sites involved in the exohedral 2e3c bond in $\gamma-\mathrm{B}_{28}$ are greater than 0.60 . Nonetheless, this observation is related to the magnitude of $V_{z z}$ and consequently to $C_{\mathrm{q}}$. In cases of large quadrupolar coupling constants where the electron density distribution around the $V_{z z}$ axis is nearly symmetric, $\eta_{\mathrm{q}}$ can be expected to approach zero as for the equatorial site in $\mathrm{B}_{12} \mathrm{O}_{2}$.

The reported insights as well as further calculations on NMR parameters of other boron-rich borides with icosahedral structure element may contribute several structure and order variants still under discussion. For example, by means of model calculations one may elucidate at which positions $\mathrm{Si}$ atoms are incorporated in $\mathrm{B}_{12}$ icosahedra of $\alpha-\mathrm{SiB}_{3-x}$ providing further knowledge to current studies. ${ }^{18}$ Besides the computation of 
NMR parameters regarding ${ }^{11} \mathrm{~B}$ we expect theoretical and experimental investigation of ${ }^{29} \mathrm{Si}$ to play a major role.

\section{Conflicts of interest}

There are no conflicts to declare.

\section{Acknowledgements}

The authors thank Dr Anke Schaub for the MAS NMR measurements.

\section{Notes and references}

1 B. Albert and H. Hillebrecht, Angew. Chem., Int. Ed., 2009, 48, 8640-8668.

2 H. C. Longuet-Higgins, Q. Rev., 1957, 11, 121.

3 H. Longuet-Higgins and M. d. V. Roberts, Proc. R. Soc. London, Ser. A, 1955, 230, 110-119.

4 K. Wade, J. Chem. Soc. D, 1971, 792-793.

5 S. Mebs, R. Kalinowski, S. Grabowsky, D. Förster, R. Kickbusch, E. Justus, W. Morgenroth, C. Paulmann, P. Luger, D. Gabel and D. Lentz, Inorg. Chem., 2011, 50, 90-103.

6 X. Jiang, J. Zhao, A. Wu, Y. Bai and X. Jiang, J. Phys.: Condens. Matter, 2010, 22, 315503.

7 V. Sagawe, PhD thesis, Albert-Ludwigs-Universität Freiburg im Breisgau, 2013.

8 H. Ozisik, E. Deligoz, K. Colakoglu and E. Ateser, Intermetallics, 2013, 39, 84-88.

9 A. R. Oganov, J. Chen, C. Gatti, Y. Ma, Y. Ma, C. W. Glass, Z. Liu, T. Yu, O. O. Kurakevych and V. L. Solozhenko, Nature, 2009, 457, 863-867.

10 E. Y. Zarechnaya, L. Dubrovinsky, N. Dubrovinskaia, Y. Filinchuk, D. Chernyshov, V. Dmitriev, N. Miyajima, A. El Goresy, H. F. Braun, S. Van Smaalen, I. Kantor, A. Kantor, V. Prakapenka, M. Hanfland, A. S. Mikhaylushkin, I. A. Abrikosov and S. I. Simak, Phys. Rev. Lett., 2009, 102, 8-11.

11 U. Häussermann and A. S. Mikhaylushkin, Inorg. Chem., 2010, 49, 11270-11275.

12 P. Macchi, J. Superhard Mater., 2011, 33, 380-387.

13 S. Mondal, S. Van Smaalen, A. Schönleber, Y. Filinchuk, D. Chernyshov, S. I. Simak, A. S. Mikhaylushkin, I. A. Abrikosov, E. Zarechnaya, L. Dubrovinsky and N. Dubrovinskaia, Phys. Rev. Lett., 2011, 106, 215502.

14 A. R. Oganov, V. L. Solozhenko, C. Gatti, O. O. Kurakevych and Y. Le Godec, J. Superhard Mater., 2011, 33, 363-379.

15 S. Mondal, S. Van Smaalen, G. Parakhonskiy, S. J. Prathapa, L. Noohinejad, E. Bykova, N. Dubrovinskaia, D. Chernyshov and L. Dubrovinsky, Phys. Rev. B: Condens. Matter Mater. Phys., 2013, 88, 1-8.

16 P. Rulis, L. Wang, B. Walker and W. Y. Ching, J. Superhard Mater., 2011, 33, 394-400.
17 S. H. Zhang, X. Zheng, Q. Q. Jin, S. J. Zheng, D. Legut, X. H. Yu, H. Y. Gou, Z. H. Fu, Y. Q. Guo, B. M. Yan, C. Peng, C. Q. Jin, T. C. Germann and R. F. Zhang, Phys. Rev. Mater., 2018, 2, 123602.

18 D. Eklöf, A. Fischer, A. Ektarawong, A. Jaworski, A. J. Pell, J. Grins, S. I. Simak, B. Alling, Y. Wu, M. Widom, W. Scherer and U. Häussermann, ACS Omega, 2019, 4, 18741-18759.

19 Y. T. Angel Wong and D. L. Bryce, Recent Advances in ${ }^{11} B$ Solid-State Nuclear Magnetic Resonance Spectroscopy of Crystalline Solids, Elsevier Ltd, 1st edn, 2018, vol. 93, pp. 213-279.

20 S. E. Ashbrook and D. McKay, Chem. Commun., 2016, 52, 7186-7204.

21 A. Samoson, Chem. Phys. Lett., 1985, 119, 29-32.

22 K. J. D. MacKenzie and M. E. Smith, Multinuclear Solid-State NMR of Inorganic Materials, Elsevier, 2002, pp. 23-108.

23 Z. Fojud, P. Herzig, O. J. Zogał, A. Pietraszko, A. Dukhnenko, S. Jurga and N. Shitsevalova, Phys. Rev. B: Condens. Matter Mater. Phys., 2007, 75, 1-5.

24 R. Schmitt, B. Blaschkowski, K. Eichele and H. J. Meyer, Inorg. Chem., 2006, 45, 3067-3073.

25 B. Jäger, S. Paluch, O. J. Zogał, W. Wolf, P. Herzig, V. B. Filippov, N. Shitsevalova and Y. Paderno, J. Phys.: Condens. Matter, 2006, 18, 2525-2535.

26 K. Schwarz, H. Ripplinger and P. Blaha, Z. Naturforsch., A: Phys. Sci., 1996, 51, 527-533.

27 H. Ripplinger, K. Schwarz and P. Blaha, J. Solid State Chem., 1997, 133, 51-54.

28 B. Jäger, S. Paluch, W. Wolf, P. Herzig, O. J. Zogał, N. Shitsevalova and Y. Paderno, J. Alloys Compd., 2004, 383, 232-238.

29 P. Herzig, Z. Fojud, O. J. Oga, A. Pietraszko, A. Dukhnenko, S. Jurga and N. Shitsevalova, J. Appl. Phys., 2008, 103, 083534.

30 C. Benndorf, M. de Oliveira, C. Doerenkamp, F. Haarmann, T. Fickenscher, J. Kösters, H. Eckert and R. Pöttgen, Dalton Trans., 2019, 48, 1118-1128.

31 S. Heřmánek, Inorg. Chim. Acta, 1999, 289, 20-44.

32 A. Lötz and J. Voitländer, J. Chem. Phys., 1991, 95, 3208-3212.

33 A. H. Silver and P. J. Bray, J. Chem. Phys., 1959, 31, 247-253.

34 D. Lee, P. J. Bray and T. L. Aselage, J. Phys.: Condens. Matter, 1999, 11, 4435-4450.

35 K. Kanehashi and K. Saito, J. Mol. Struct., 2002, 602-603, 105-113.

36 F. A. Perras and D. L. Bryce, J. Chem. Phys., 2013, 138, 174202.

37 C. J. Pickard and F. Mauri, Phys. Rev. B: Condens. Matter Mater. Phys., 2001, 63, 245101.

38 J. R. Yates, C. J. Pickard and F. Mauri, Phys. Rev. B: Condens. Matter Mater. Phys., 2007, 76, 024401.

39 M. Ludwig, D. Himmel and H. Hillebrecht, J. Phys. Chem. A, 2020, 124, 2173-2185.

40 M. Ludwig and H. Hillebrecht, Phys. Chem. Chem. Phys., 2021, 23, 470-486.

41 A. Pediaditakis, M. Schroeder, V. Sagawe, T. Ludwig and H. Hillebrecht, Inorg. Chem., 2010, 49, 10882-10893. 
42 V. Adasch, K. U. Hess, T. Ludwig, N. Vojteer and H. Hillebrecht, Chem. - Eur. J., 2007, 13, 3450-3458.

43 R. Bader, Atoms in Molecules: A Quantum Theory, Clarendon Press, 1994.

44 P. Giannozzi, S. Baroni, N. Bonini, M. Calandra, R. Car, C. Cavazzoni, D. Ceresoli, G. L. Chiarotti, M. Cococcioni, I. Dabo, A. D. Corso, S. de Gironcoli, S. Fabris, G. Fratesi, R. Gebauer, U. Gerstmann, C. Gougoussis, A. Kokalj, M. Lazzeri, L. Martin-Samos, N. Marzari, F. Mauri, R. Mazzarello, S. Paolini, A. Pasquarello, L. Paulatto, C. Sbraccia, S. Scandolo, G. Sclauzero, A. P. Seitsonen, A. Smogunov, P. Umari and R. M. Wentzcovitch, J. Phys.: Condens. Matter, 2009, 21, 395502.

45 P. Giannozzi, O. Andreussi, T. Brumme, O. Bunau, M. B. Nardelli, M. Calandra, R. Car, C. Cavazzoni, D. Ceresoli, M. Cococcioni, N. Colonna, I. Carnimeo, A. D. Corso, S. de Gironcoli, P. Delugas, R. A. Delugas Jr, A. Ferretti, A. Floris, G. Fratesi, G. Fugallo, R. Gebauer, U. Gerstmann, F. Giustino, T. Gorni, J. Jia, M. Kawamura, H.-Y. Ko, A. Kokalj, E. Küçükbenli, M. Lazzeri, M. Marsili, N. Marzari, F. Mauri, N. L. Nguyen, H.-V. Nguyen, A. Oterode-la Roza, L. Paulatto, S. Poncé, D. Rocca, R. Sabatini, B. Santra, M. Schlipf, A. P. Seitsonen, A. Smogunov, I. Timrov, T. Thonhauser, P. Umari, N. Vast, X. Wu and S. Baroni, J. Phys.: Condens. Matter, 2017, 29, 465901.

46 J. Perdew, K. Burke and M. Ernzerhof, Phys. Rev. Lett., 1996, 77, 3865-3868.

47 T. Björkman, Comput. Phys. Commun., 2011, 182, 1183-1186.

48 H. J. Monkhorst and J. D. Pack, Phys. Rev. B: Solid State, 1976, 13, 5188-5192.

49 C. Hartwigsen, S. Goedecker and J. Hutter, Phys. Rev. B: Condens. Matter Mater. Phys., 1998, 58, 3641-3662.

50 N. Troullier and J. L. Martins, Phys. Rev. B: Condens. Matter Mater. Phys., 1991, 43, 1993-2006.

51 G. Kresse and D. Joubert, Phys. Rev. B: Condens. Matter Mater. Phys., 1999, 59, 1758-1775.

52 R. K. Harris, E. D. Becker, S. M. Cabral De Menezes, P. Granger, R. E. Hoffman and K. W. Zilm, Pure Appl. Chem., 2008, 80, 59-84.

53 M. Bak, J. T. Rasmussen and N. C. Nielsen, J. Magn. Reson., 2000, 147, 296-330.

54 P. Pyykkö, Mol. Phys., 2018, 116, 1328-1338.

55 A. Otero-de-la Roza, M. A. Blanco, A. M. Pendás and V. Luaña, Comput. Phys. Commun., 2009, 180, 157-166.

56 A. Otero-de-la Roza, E. Johnson and V. Luaña, Comput. Phys. Commun., 2014, 185, 1007-1018.
57 D. Massiot, F. Fayon, M. Capron, I. King, S. Le Calvé, B. Alonso, J.-O. Durand, B. Bujoli, Z. Gan and G. Hoatson, Magn. Reson. Chem., 2002, 40, 70-76.

58 K. Momma and F. Izumi, J. Appl. Crystallogr., 2011, 44, 1272-1276.

59 R. F. Bader, T. S. Slee, D. Cremer and E. Kraka, J. Am. Chem. Soc., 1983, 105, 5061-5068.

60 In context of our previous investigation ${ }^{40}$ we found an ellipticity value $\varepsilon=3.84$ for the exohedral $2 \mathrm{e} 3 \mathrm{c}$ bond in $\alpha-\mathrm{rB}_{12}$.

61 R. Lortz, Y. Wang, U. Tutsch, S. Abe, C. Meingast, P. Popovich, W. Knafo, N. Shitsevalova, Y. B. Paderno and A. Junod, Phys. Rev. B: Condens. Matter Mater. Phys., 2006, 73, 024512.

62 O. J. Zogał, P. Florian, D. Massiot, S. Paluch, N. Shitsevalova and D. F. Borshchevsky, Solid State Commun., 2009, 149, 693-696.

63 K. H. J. Buschow and J. H. N. Creyghton, J. Chem. Phys., 1972, 57, 3910-3914.

64 F. Mauri, B. G. Pfrommer and S. G. Louie, Phys. Rev. Lett., 1996, 77, 5300-5303.

65 M. D’Avezac, N. Marzari and F. Mauri, Phys. Rev. B: Condens. Matter Mater. Phys., 2007, 76, 1-12.

66 T. Thonhauser, D. Ceresoli, A. A. Mostofi, N. Marzari, R. Resta and D. Vanderbilt, J. Chem. Phys., 2009, 131, 101101.

67 P. J. Basser and C. Pierpaoli, J. Magn. Reson., 1996, 111, 209-219.

68 E. Özarslan, B. C. Vemuri and T. H. Mareci, Magn. Reson. Med., 2005, 53, 866-876.

69 For $\alpha-\mathrm{rB}_{12}$ we obtain $\mathrm{fa}_{\chi}=48.1 \%$ rising from the deviation between the isotropic value $\bar{\chi}$ and the $\chi_{\text {bare }}$ component oriented to the exohedral $2 \mathrm{e} 3 \mathrm{c}$ bond.

70 Z. P. Yin and W. E. Pickett, Phys. Rev. B: Condens. Matter Mater. Phys., 2008, 77, 035135.

71 R. Laskowski and P. Blaha, J. Phys. Chem. C, 2015, 119, 19390-19396.

72 A. R. Ferreira, K. Reuter and C. Scheurer, J. Phys. Chem. C, 2016, 120, 25530-25540.

73 K. H. Khoo, R. Laskowski and P. Blaha, J. Phys. Chem. C, 2017, 121, 12398-12406.

74 Using the geometry reported in ref. 39.

75 For $\mathrm{B}(3)$ in $\gamma-\mathrm{B}_{28}$ its distance $d_{\mathrm{X}-\mathrm{BCP}}$ to the $\mathrm{BCP}$ in the $\mathrm{B}(2)-$ $\mathrm{B}(5)$ direction (see also Fig. 8) with $1.674 \AA$ is chosen.

76 M. Profeta, M. Benoit, F. Mauri and C. J. Pickard, J. Am. Chem. Soc., 2004, 126, 12628-12635. 